\title{
Optimal Reinsurance-Investment Problem under a CEV Model: Stochastic Differential Game Formulation
}

\author{
Danping Li $\left(\mathbb{D},{ }^{1}\right.$ Ruiqing Chen, ${ }^{1}$ and Cunfang $\mathrm{Li}$ (D) ${ }^{2}$ \\ ${ }^{1}$ Key Laboratory of Advanced Theory and Application, Statistics and Data Science-MOE, School of Statistics, \\ East China Normal University, Shanghai 200062, China \\ ${ }^{2}$ School of Business, Jiangsu Normal University, Xuzhou, Jiangsu 221000, China \\ Correspondence should be addressed to Danping Li; dpli@fem.ecnu.edu.cn
}

Received 18 February 2020; Accepted 14 March 2020; Published 21 April 2020

Guest Editor: Wenguang Yu

Copyright (C) 2020 Danping Li et al. This is an open access article distributed under the Creative Commons Attribution License, which permits unrestricted use, distribution, and reproduction in any medium, provided the original work is properly cited.

\begin{abstract}
This paper focuses on a stochastic differential game played between two insurance companies, a big one and a small one. In our model, the basic claim process is assumed to follow a Brownian motion with drift. Both of two insurance companies purchase the reinsurance, respectively. The big company has sufficient asset to invest in the risky asset which is described by the constant elasticity of variance (CEV) model and acquire new business like acting as a reinsurance company of other insurance companies, while the small company can invest in the risk-free asset and purchase reinsurance. The game studied here is zero-sum where there is a single exponential utility. The big company is trying to maximize the expected exponential utility of the terminal wealth to keep its advantage on surplus while simultaneously the small company is trying to minimize the same quantity to reduce its disadvantage. In this paper, we describe the Nash equilibrium of the game and prove a verification theorem for the exponential utility. By solving the corresponding Fleming-Bellman-Isaacs equations, we derive the optimal reinsurance and investment strategies. Furthermore, numerical examples are presented to show our results.
\end{abstract}

\section{Introduction}

Recently, most insurance companies manage their business by means of reinsurance and investment, which are effective way to spread risk and make profit. Therefore, these have inspired hundred researches. For instance, Schmidli [1], Promislow and Young [2], and Bai and Guo [3] investigated the optimal problems for an insurance company in the case of minimizing the ruin probability. Yang and Zhang [4], Wang [5], and Cao and Wan [6] studied the optimal reinsurance and investment problems of expected utility maximization. The latest researches on insurance and investment management problem can be referred to $\mathrm{Yu}$ et al. [7], Zhang et al. [8], Peng et al. [9], Yu et al. [10], Ruan et al. [11], Yu et al. [12], Huang et al. [13], Zeng et al. [14], Li et al. [15] and references therein.

However, most of the literature mentioned above only considered one insurance company, while there are many insurance companies in the market in reality and they compete with each other. Thus, two insurance companies, a big one and a small one, are focused on in this paper. This competition between the two insurance companies can be formulated as a stochastic differential game. In previous researches, Suijs et al. [16] showed that problems in non-life insurance and non-life reinsurance can be modeled as cooperative games. Zeng [17] discussed the competition between two companies and contrasted a single payoff function which depended on both insurance companies' surplus processes. Taksar and Zeng [18] investigated stochastic differential games between two insurance companies who employed the reinsurance to reduce risk exposure. Some papers focus on the relative performance of two insurance companies under a nonzero sum stochastic differential game framework, such as Bensoussan et al. [19], Meng et al. [20], Pun and Wong [21], and Siu et al. [22]. However, some literatures related to stochastic differential games ignore the problem of investment in insurance companies. Nowadays, investment plays a significant role in the insurance business, 
especially for those big insurance companies who have enough ability to invest in the risky asset for more profits.

Another aspect worthy to be further explored is that the price processes of risky assets in most of literature about the optimal reinsurance and investment problems in frameworks of stochastic differential games are assumed to follow a geometric Brownian motion (GBM), which implies that the volatilities of risky assets are constant and deterministic. This is contrary to practice according to the empirical results. Therefore, many works proposed various stochastic volatility models, such as constant elasticity of variance (CEV) model (Cox and Ross [23]), Stein-Stein model (Stein and Stein [24]), Heston model (Heston [25]) and so on. Among these stochastic volatility models, the CEV model is a natural extension of the GBM model and has the ability of capturing the implied volatility skew and explaining the volatility smile. There is a great deal of literature documenting the CEV model in assets' return for the optimal investment problem. For example, Gu et al. [26] considered the proportional reinsurance and investment problem for the diffusion risk model under the CEV model. Liang et al. [27] and Lin and Li [28] used the CEV model to study the proportional reinsurance and investment problem for an insurance company with the jump-diffusion risk model. Gu et al. [29] derived the excess-of-loss reinsurance and investment strategies with the risky asset's price following the CEV model. Zheng et al. [30] considered the robust optimal portfolio and proportional reinsurance for an insurer under a CEV model.

As far as we know, there is few research investigating more than one insurance company under the CEV model. Therefore, in this paper, we consider a stochastic differential game played between two insurance companies, a big one and a small one. In our model, the basic claim processes is assumed to follow a Brownian motion with drift. Both of two insurance companies purchase the reinsurance, respectively. The big insurance company has more initial surplus than the small one, so the big company has sufficient asset to invest in the risky asset which is described by the CEV model and acquire new business like acting as a reinsurance company of other insurance companies, while the small company can only invest in the risk-free asset and purchase reinsurance. The game studied here is zero-sum where there is a single exponential utility. The big company is trying to maximize the expected exponential utility of the terminal wealth to keep its advantage on surplus while simultaneously the small company is trying to minimize the same quantity to reduce its disadvantage. Firstly, we describe the Nash equilibrium of the game and prove a verification theorem for the exponential utility. By solving the corresponding Fleming-Bellman-Isaacs equations, we derive the optimal reinsurance and investment strategies. Finally, numerical simulation are proposed to illustrate the impacts of the model parameters on the strategies. Through this paper, we find that (1) for such a game the small insurance company takes extreme or trivial strategy, i.e., the optimal reinsurance strategy of the small company is either 1 or 0 ; (2) the optimal reinsurance and investment strategies of the big company are independent of the wealth process $X^{u_{1}, u_{2}}(t)$; (3) the effects of some model parameters on the big company's optimal reinsurance strategy are related to the correlation coefficient between the big and small companies' risk processes.

This paper proceeds as follows. In Section 2, we introduce the formulation of our model, describe the Nash equilibrium of the game, and prove a verification theorem for the exponential utility under the CEV model. Section 3 provides the optimal reinsurance and investment strategies of the big insurance company and the optimal reinsurance strategy of the small one in the stochastic differential game. In Section 4, numerical simulations are presented to illustrate our results. Section 5 concludes this paper.

\section{Model Formulation}

In this paper, we model the surplus process of the insurance company as

$$
\mathrm{d} R(t)=a \mathrm{~d} t+b \mathrm{~d} W(t)
$$

where $a, b$ are positive constants and $W(t)$ is a standard Brownian motion. To reduce the risk exposure, the insurance company is allowed to purchase the reinsurance and $p(t)$ represents the proportion of each claim paid by the insurance company. Assume $\lambda(1-p(t))$ is the rate at which the premiums are diverted to the reinsurance company. As usual, we have $\lambda \geq a$. Otherwise the insurance company will make a full reinsurance to receive a positive return with any risk. Considering the reinsurance, the surplus process of the insurance company becomes

$$
\mathrm{d} R(t)=(a-(1-p(t)) \lambda) \mathrm{d} t+b p(t) \mathrm{d} W(t) .
$$

In this paper, we consider a stochastic differential game played between two insurance companies, a big one and a small one. The big insurance company has more initial surplus than the small one, so the big company has sufficient asset to invest in the risky asset and acquire new business like acting as a reinsurance company of other insurance companies, while the small company can only invest in the risk-free asset and purchase reinsurance, i.e., the reinsurance strategies of the big and small companies $p_{1}(t)$ and $p_{2}(t)$ satisfy $0 \leq p_{1}(t), 0 \leq p_{2}(t) \leq 1$. The game considered here is zero-sum where there is a single exponential utility. The big company is trying to maximize the expected exponential utility of the terminal wealth to keep its advantage on surplus while simultaneously the small company is trying to minimize the same quantity to reduce its disadvantage. One company's decision is assumed to be completely observed by its opponent.

Let $\left(\Omega, \mathscr{F},\left(\mathscr{F}_{t}\right), P\right)$ be a complete probability space with filtration $\left(\mathscr{F}_{t}\right)$ and two standard Brownian motions $W_{1}(t)$ and $W_{2}(t)$, adapted to $\left(\mathscr{F}_{t}\right)$ with $\mathscr{F}=\mathscr{F}_{T}$, where $T$ is a fixed and finite time horizon. The surplus processes of the two insurance companies associated with the proportional reinsurance $p_{i}(t), i=1,2$ are given by

$$
\mathrm{d} R_{i}(t)=\left(a_{i}\left(1-p_{i}(t)\right) \lambda_{i}\right) \mathrm{d} t+b_{i} p_{i}(t) \mathrm{d} W_{i}(t), \quad i=1,2,
$$

where $\lambda_{i} \geq a_{i}>0, b_{i}>0, i=1,2$ are constants, $E\left[W_{1}(t) W_{2}\right.$ $(t)]=\rho_{12} t$. 
In this paper, the big insurance company is allowed to invest in a risk-free asset whose price process satisfies

$$
\mathrm{d} S_{0}(t)=r_{0} S_{0}(t) \mathrm{d} t, \quad S_{0}(0)=1,
$$

and a risky asset whose price process is described by the CEV model (cf. Cox [31]):

$$
\mathrm{d} S(t)=S(t)\left(\mu \mathrm{d} t+\sigma S^{\beta}(t) \mathrm{d} W_{3}(t)\right),
$$

$S(0)=s_{0}$, where $r_{0}$ is the interest rate and $\mu, \sigma S^{\beta}(t)$, and $\beta$ are the appreciation rate, the instantaneous volatility, and the elasticity parameter of the risky asset. $W_{3}(t)$ is a standard Brownian motion independent of $W_{1}(t)$ and $W_{2}(t)$. As usual, we assume that $\mu>r_{0}$ and $\beta$ satisfies the general condition $\beta \geq 0$. Meanwhile, the small one can invest in a risk-free asset to avoid risk. Strategies $u_{1}:=\left(\pi(t), p_{1}(t)\right)$ and $u_{2}:=p_{2}(t)$ are said to be admissible if they are $\left(\mathscr{F}_{t}\right)$-progressively measurable and satisfy $\left(u_{1}, u_{2}\right):=\left(\left(\pi(t), p_{1}(t)\right), p_{2}(t)\right) \in \Pi:$

$$
\begin{aligned}
\Pi= & \left\{\left(u_{1}, u_{2}\right): E\left[\int_{0}^{T} \pi^{2}(t) \mathrm{d} t\right]<\infty, E\left[\int_{0}^{T} p_{1}^{2}(t) \mathrm{d} t\right]<\infty,\right. \\
& \left.0 \leq p_{1}(t), 0 \leq p_{2}(t) \leq 1\right\},
\end{aligned}
$$

where $\pi(t)$ represents the amount invested in the risky asset by the big insurance company at time $t$. Here $\Pi$ is called the admissible set. Corresponding to an admissible strategy and the initial wealth $x_{1}>x_{2}$, the wealth processes of the big and small insurance companies $X_{1}^{u_{1}}(t)$ and $X_{2}^{u_{1}}(t)$ are

$$
\begin{aligned}
& \left\{\begin{array}{l}
\mathrm{d} X_{1}^{u_{1}}(t)=\left[r_{0} X_{1}^{u_{1}}(t)+\pi(t)\left(\mu-r_{0}\right)+a_{1}-\left(1-p_{1}(t)\right) \lambda_{1}\right] \mathrm{d} t+\pi(t) \sigma S^{\beta}(t) \mathrm{d} W_{3}(t)+b_{1} p_{1}(t) \mathrm{d} W_{1}(t) \\
X_{1}^{u_{1}}(0)=x_{1}
\end{array}\right. \\
& \left\{\begin{array}{l}
\mathrm{d} X_{2}^{u_{2}}(t)=\left[r_{0} X_{2}^{u_{2}}(t)+a_{2}-\left(1-p_{2}(t)\right) \lambda_{2}\right] \mathrm{d} t+b_{2} p_{2}(t) \mathrm{d} W_{2}(t) \\
X_{2}^{u_{2}}(0)=x_{2}
\end{array}\right.
\end{aligned}
$$

Let $X^{u_{1}, u_{2}}(t)=X_{1}^{u_{1}}(t)-X_{2}^{u_{2}}(t)$; then $X^{u_{1}, u_{2}}(t)$ follows the following stochastic differential equation:

$$
\left\{\begin{array}{l}
\mathrm{d} X^{u_{1}, u_{2}}(t)=\left[r_{0} X^{u_{1}, u_{2}}(t)+\pi(t)\left(\mu-r_{0}\right)+D+p_{1}(t) \lambda_{1}-p_{2}(t) \lambda_{2}\right] \mathrm{d} t+\pi(t) \sigma S^{\beta}(t) \mathrm{d} W_{3}(t) \\
\quad+b_{1} p_{1}(t) \mathrm{d} W_{1}(t)-b_{2} p_{2}(t) \mathrm{d} W_{2}(t), \\
X^{u_{1}, u_{2}}(0)=x_{1}-x_{2}=x,
\end{array}\right.
$$

where $D=a_{1}-\lambda_{1}-\left(a_{2}-\lambda_{2}\right)$. Here, we usually assume $x>0$, which describes that one of the two insurance companies is big, and the other is small. That is, this model is suitable for the case that $x$ is positive and we can distinguish the big and small companies easily. If $x$ is very small, we will choose the insurance company with larger initial wealth as the big one, and the other is the small one. If it is difficult to distinguish the big company and small company from the initial wealth, this framework may not be suitable.

Remark 1 . If the elasticity parameter $\beta=0$ in equation (1), the CEV model reduces to the GBM model.

In this paper, we consider the exponential utility which is given by

$$
U(x)=-\frac{1}{\gamma} \mathrm{e}^{-\gamma x},
$$

where $\gamma>0$. The exponential utility has the constant absolute risk aversion parameter $\gamma$, which plays an important role in insurance mathematics and actuarial practice.
Definition 1. Let

$$
J^{u_{1}, u_{2}}(t, s, x)=\mathrm{E}\left[U\left(X^{u_{1}, u_{2}}(T)\right) \mid S(t)=s, X^{u_{1}, u_{2}}(t)=x\right],
$$

the strategy $\left(u_{1}^{*}, u_{2}^{*}\right)$ is said to achieve a Nash equilibrium or equivalently a saddle point for the game, if the following inequalities are satisfied. For all $\left(u_{1}, u_{2}\right) \in \Pi$,

$$
J^{u_{1}, u_{2}^{*}}(t, s, x) \leq J^{u_{1}^{*}, u_{2}^{*}}(t, s, x) \leq J^{u_{1}^{*}, u_{2}}(t, s, x) .
$$

In addition, let

$$
\begin{aligned}
& \underline{J}(t, s, x)=\sup _{u_{2} \in \Pi} \inf _{u_{2} \in \Pi} J^{u_{1}, u_{2}}(t, s, x), \\
& \bar{J}(t, s, x)=\inf _{u_{2} \in \Pi} \sup _{u_{1} \in \Pi} J^{u_{1}, u_{2}}(t, s, x),
\end{aligned}
$$

denote the lower and upper values of the game respectively. If $J(t, s, x)=\bar{J}(t, s, x)$, the value function of the game is given by $G(x):=J(t, s, x)=\bar{J}(t, s, x)$.

In this paper, the goal of the big insurance company is to maximize the above expected exponential utility function while simultaneously the small one wants to minimize it. For 
convenience, we first provide some notations. Let $\mathrm{O}_{0} \subset \mathbb{R}^{2}$

be an open set and $O=[0, T] \times O_{0}$. Denote that

$$
\begin{aligned}
C^{1,2}(O)= & \{\phi(t, s, x) \mid \phi(t, \cdot, \cdot) \text { is once continuously differentiable on }[0, T] \\
& \text { and } \left.\phi(\cdot, s, x) \text { is twice continuously differentiable on } O_{0}\right\}
\end{aligned}
$$

and define a variation operator: for any $\phi(t, s, x) \in C^{1,2}(O)$,

let

$$
\begin{aligned}
\mathscr{A}^{u_{1}, u_{2}} \phi(t, s, x)= & \phi_{t}+\left[r_{0} x+\pi\left(\mu-r_{0}\right)+D+p_{1} \lambda_{1}-p_{2} \lambda_{2}\right] \phi_{x}+\frac{1}{2}\left[\pi^{2} \sigma^{2} s^{2 \beta}+p_{1}^{2} b_{1}^{2}\right. \\
& \left.+p_{2}^{2} b_{2}^{2}-2 p_{1} p_{2} b_{1} b_{2} \rho_{12}\right] \phi_{x x}+\mu s \phi_{s}+\frac{1}{2} \sigma^{2} s^{2 \beta+2} \phi_{s s}+\pi \sigma^{2} s^{2 \beta+1} \phi_{x s} .
\end{aligned}
$$

For any given strategy $u_{2}$ by the small insurance company, let $\underline{V}^{u_{2}}(t, s, x)$ be the optimal expected exponential utility function of the big insurance company, i.e.,

$$
\underline{V}(t, s, x)=\sup _{u_{1} \in \Pi} J^{u_{1}, u_{2}}(t, s, x) .
$$

Then $\underline{V}^{u_{2}}(t, s, x)$ satisfies the following Hamilton-JacobiBellman (HJB) equation

$$
\sup _{u_{1} \in \Pi} A^{u_{1}, u_{2}} \underline{V}^{u_{2}}(t, s, x)=0, \quad 0 \leq t \leq T .
$$

Similarly, let $\bar{V}^{u_{1}}(t, s, x)$ be the optimal expected exponential utility function of the small insurance company with any strategy $u_{1}$ given by the big insurance company, then $\bar{V}^{u_{1}}(t, s, x)$ satisfied another HJB equation

$$
\inf _{u_{2} \in \Pi} \mathscr{A}^{u_{1}, u_{2}} \bar{V}^{u_{1}}(t, s, x)=0, \quad 0 \leq t \leq T .
$$

Denote

$$
\begin{aligned}
& \widehat{u_{1}}:=\arg \sup _{u_{1} \in \Pi} \mathscr{A}^{u_{1}, u_{2}} \underline{\underline{V}}(t, s, x), \\
& \widehat{u_{2}}:=\arg \inf _{u_{2} \in \Pi} \mathscr{A}^{u_{1}, u_{2}} \bar{V}^{u_{1}}(t, s, x) .
\end{aligned}
$$

Assume that a saddle point exits, then the game have a value function ${ }_{-} V(t, s, x)=V^{u_{1}, u_{2}}(t, s, x)=V^{u_{1}, u_{2}}(t, s, x)=$ $\underline{V}^{\widehat{u}_{2}}(t, s, x)=\bar{V}^{u_{1}}(t, s, x)$.

Let $\left(u_{1}^{*}, u_{2}^{*}\right)$ be the solution to the following equations:

$$
\begin{aligned}
& \widehat{u_{1}}:=\arg \sup _{u_{1} \in \Pi} \mathscr{A}^{u_{1}, \widehat{u_{2}}} \underline{\underline{u_{2}}}(t, s, x), \\
& \widehat{u_{2}}:=\arg \inf _{u_{2} \in \Pi} \mathscr{A}^{\widehat{u_{1}}, u_{2}} \bar{V}^{\widehat{u}_{1}}(t, s, x),
\end{aligned}
$$

and substitute them into equations (16) and (17), respectively, we obtain the following equations:

$$
\begin{array}{ll}
\sup _{u_{1} \in \Pi} \mathscr{A}^{u_{1}, u_{2}^{*}} V^{u_{1}, u_{2}^{*}}(t, s, x)=0, & 0 \leq t \leq T, \\
\inf _{u_{2} \in \Pi} \mathscr{A}^{u_{1}^{*}, u_{2}} V^{u_{1}^{*}, u_{2}}(t, s, x)=0, & 0 \leq t \leq T,
\end{array}
$$

with the boundary condition $V(T, s, x)=U(x)$.

Theorem 1 (Verification theorem). If there exists a continuously differential function $H(t, s, x) \in C^{1,2}(O)$ and

$$
\begin{aligned}
& u_{1}^{*}:=\arg \sup _{u_{1} \in \Pi} \mathscr{A}^{u_{1}, u_{2}^{*}} H^{u_{1}, u_{2}^{*}}(t, s, x), \\
& u_{2}^{*}:=\arg \inf _{u_{2} \in \Pi} \mathscr{A}^{u_{1}^{*}, u_{2}} H^{u_{1}^{*}, u_{2}}(t, s, x),
\end{aligned}
$$

satisfy equations (20) and (21) with the following moment properties

$$
\begin{aligned}
& \int_{0}^{t} E\left[H_{s}^{2}(\nu, s, x)\right] \mathrm{d} \nu<\infty, \\
& \int_{0}^{t} E\left[H_{x}^{2}[\nu, s, x]\right] \mathrm{d} \nu<\infty .
\end{aligned}
$$

In addition, the parameters satisfy one of the following conditions:

(a) $r_{0}>(1-(1 / \sqrt{6})) \mu$;

(b) $r_{0}<(1-(1 / \sqrt{6})) \mu T<\left(1 /\left(\beta \sqrt{6\left(\mu-r_{0}\right)^{2}-\mu^{2}}\right)\right) \arctan$ $\left(-\left(\left(\sqrt{6\left(\mu_{2}-r_{0}\right)^{2}-\mu_{2}^{2}}\right) / \mu_{2}\right)\right)$, then $\left(u_{1}^{*}, u_{2}^{*}\right)$ is the optimal strategy and the optimal value function is $V(t, s, x)=H(t, s, x)$.

Proof. See Appendix A.

The above theorem guarantees the solution to equations (20) and (21) is the value function for the game.

\section{Solution to the Model}

In this section, we solve the game under the expected exponential utility. The big company is trying to maximize the expected exponential utility of the terminal wealth to keep its advantage on surplus while the small company is trying to minimize the same quantity to reduce its disadvantage, i.e., 


$$
J^{u_{1}, u_{2}}(t, s, x)=E\left[U\left(X^{u_{1}, u_{2}}(T)\right) \mid S(t)=s, X^{u_{1}, u_{2}}(t)=x\right] .
$$

Assume the Nash equilibrium exits and let $H(t, s, x)$ satisfy

$$
H(t, s, x)=\sup _{u_{1} \in \Pi} \inf _{u_{2} \in \Pi} J^{u_{1}, u_{2}}(t, s, x)=\inf _{u_{2} \in \Pi} \sup _{u_{1} \in \Pi} J^{u_{1}, u_{2}}(t, s, x),
$$

then $H(t, s, x)$ is the solution to equations (20) and (21) with the boundary condition $H(T, s, x)=(x)$ and $H(t, s, x)$ is the optimal value function according to Theorem 1 .

The following theorem gives the optimal reinsurance and investment strategies of the big company and the reinsurance strategy of the small company in the game under the CEV model with the expected exponential utility.

Theorem 2. For the problem of maximizing the expected exponential utility for the big company while minimizing it for the small company under the CEV model, the optimal reinsurance and investment strategies are given as follows:

(1) If $\left(\left(\lambda_{2} b_{1}-\rho_{12} \lambda_{1} b_{2}\right) /\left(b_{1} b_{2}^{2}\left(1-\rho_{12}^{2}\right)\right)\right) \geq-(\gamma / 2)$ and $\rho_{12} \geq 0$, the optimal reinsurance and investment strategies of the big company and the reinsurance strategy of the small company are

$$
\begin{array}{r}
\left(p_{1}^{*}(t), p_{2}^{*}(t)\right)=\left(\frac{\lambda_{1}}{\gamma b_{1}^{2}} \mathrm{e}^{-r_{0}(T-t)}+\frac{b_{2} \rho_{12}}{b_{1}}, 1\right), \quad 0 \leq t \leq T, \\
(26) \\
\pi^{*}(t)=\frac{\left(\mu-r_{0}\right) \mathrm{e}^{-r_{0}(T-t)}}{\sigma^{2} s^{2 \beta} \gamma}\left[1+\frac{\mu-r_{0}}{2 r_{0}}\left(1-\mathrm{e}^{-2 r_{0} \beta(T-t)}\right)\right], \\
0 \leq t \leq T .
\end{array}
$$

The value function is given by equation (B.21).

(2) If $\left(\left(\lambda_{2} b_{1}-\rho_{12} \lambda_{1} b_{2}\right) /\left(b_{1} b_{2}^{2}\left(1-\rho_{12}^{2}\right)\right)\right) \geq-(\gamma / 2), \rho_{12}$ $<0$ and $-\left(\lambda_{1} / \gamma b_{1} b_{2} \rho_{12}\right) \leq 1$, the optimal reinsurance strategies of the big and small companies are

$$
\left(p_{1}^{*}(t), p_{2}^{*}(t)\right)=(0,1), \quad 0 \leq t \leq T,
$$

while the optimal investment strategy of the big company is the same as that in equation (11) and the value function is given in equation (B.25).

(3) If $\quad\left(\left(\lambda_{2} b_{1}-\rho_{12} \lambda_{1} b_{2}\right) /\left(b_{1} b_{2}^{2}\left(1-\rho_{12}^{2}\right)\right)\right) \geq-(\gamma / 2)$, $\rho_{12}<0, \quad-\left(\lambda_{1} / \gamma b_{1} b_{2} \rho_{12}\right)>1$ and $e^{r_{0} T}<-\left(\lambda_{1} / \gamma b_{1} b_{2}\right.$ $\left.\rho_{12}\right)$, the optimal reinsurance strategies of the big and small companies are the same as those in equation (26). The optimal investment strategy of the big company is expressed as that in equation (27) and the value function is given by equation (B.20).

(4) If $\left(\left(\lambda_{2} b_{1}-\rho_{12} \lambda_{1} b_{2}\right) /\left(b_{1} b_{2}^{2}\left(1-\rho_{12}^{2}\right)\right)\right) \geq-(\gamma / 2), \rho_{12}$ $<0,-\left(\lambda_{1} / \gamma b_{1} b_{2} \rho_{12}\right)>1$, and $e^{r_{0} T} \geq-\left(\lambda_{1} / \gamma b_{1} b_{2} \rho_{12}\right)$, the optimal reinsurance strategies of the big and small companies are

$$
\left(p_{1}^{*}(t), p_{2}^{*}(t)\right)= \begin{cases}(0,1), & 0 \leq t \leq t_{1}, \\ \left(\frac{\lambda_{1}}{\gamma b_{1}^{2}} e^{-r_{0}(T-t)}+\frac{b_{2} \rho_{12}}{b_{1}}, 1\right), & t_{1}<t \leq T,\end{cases}
$$

while the optimal investment strategy of the big company is the same as that in equation (11) and the value function is given in equation (B.28).

(5) If $\left(\left(\lambda_{2} b_{1}-\rho_{12} \lambda_{1} b_{2}\right) /\left(b_{1} b_{2}^{2}\left(1-\rho_{12}^{2}\right)\right)\right)<-(\gamma / 2)$ and $e^{r_{0} T}<-\left(2\left(\lambda_{2} b_{1}-\rho_{12} \lambda_{1} b_{2}\right) /\left(\gamma b_{1} b_{2}^{2}\left(1-\rho_{12}^{2}\right)\right)\right)$, the optimal reinsurance strategies of the big and small companies are

$\left(p_{1}^{*}(t), p_{2}^{*}(t)\right)=\left(\frac{\lambda_{1}}{\gamma b_{1}^{2}} \mathrm{e}^{-r_{0}(T-t)}, 0\right), \quad 0 \leq t \leq T$.

The optimal investment strategy of the big company is expressed as that in equation (11) and the value function is given by equation (B.32).

(6) If $\quad\left(\left(\lambda_{2} b_{1}-\rho_{12} \lambda_{1} b_{2}\right) /\left(b_{1} b_{2}^{2}\left(1-\rho_{12}^{2}\right)\right)\right)<-(\gamma / 2)$, $e^{r_{0} T} \geq-\left(2\left(\lambda_{2} b_{1}-\rho_{12} \lambda_{1} b_{2}\right) /\left(\gamma b_{1} b_{2}^{2}\left(1-\rho_{12}^{2}\right)\right)\right)$ and $\rho_{12} \geq 0$, the optimal reinsurance strategies of the big and small companies are

$$
\left(p_{1}^{*}(t), p_{2}^{*}(t)\right)= \begin{cases}\left(\frac{\lambda_{1}}{\gamma b_{1}^{2}} \mathrm{e}^{-r_{0}(T-t)}+\frac{b_{2} \rho_{12}}{b_{1}}, 1\right), & 0 \leq t \leq t_{2}, \\ \left(\frac{\lambda_{1}}{\gamma b_{1}^{2}} \mathrm{e}^{-r_{0}(T-t)}, 0\right), & t_{2}<t \leq T,\end{cases}
$$

while the optimal investment strategy of the big company is the same as that in equation (11) and the value function is given in equation (B.36).

(7) If $\quad\left(\left(\lambda_{2} b_{1}-\rho_{12} \lambda_{1} b_{2}\right) /\left(b_{1} b_{2}^{2}\left(1-\rho_{12}^{2}\right)\right)\right)<-(\gamma / 2)$, $e^{r_{0} T} \geq-\left(2\left(\lambda_{2} b_{1}-\rho_{12} \lambda_{1} b_{2}\right) /\left(\gamma b_{1} b_{2}^{2}\left(1-\rho_{12}^{2}\right)\right)\right)$, $\rho_{12}<0$ and $-\left(\lambda_{1} / \gamma b_{1} b_{2} \rho_{12}\right) \leq 1$, the optimal reinsurance strategies of the big and small companies are

$$
\left(p_{1}^{*}(t), p_{2}^{*}(t)\right)= \begin{cases}(0,1), & 0 \leq t \leq t_{2}, \\ \left(\frac{\lambda_{1}}{\gamma b_{1}^{2}} \mathrm{e}^{-r_{0}(T-t)}, 0\right), & t_{2}<t \leq T .\end{cases}
$$

Se optimal investment strategy of the big company is expressed as that in equation (11) and the value function is given by equation (B.39).

(8) If $\quad\left(\left(\lambda_{2} b_{1}-\rho_{12} \lambda_{1} b_{2}\right) /\left(b_{1} b_{2}^{2}\left(1-\rho_{12}^{2}\right)\right)\right)<-(\gamma / 2)$, $e^{r_{0} T} \geq-\left(2\left(\lambda_{2} b_{1}-\rho_{12} \lambda_{1} b_{2}\right) /\left(\gamma b_{1} b_{2}^{2}\left(1-\rho_{12}^{2}\right)\right)\right), \rho_{12}<$ $0,-\left(\lambda_{1} / \gamma b_{1} b_{2} \rho_{12}\right)>1$, and $e^{r_{0} T}<-\left(\lambda_{1} / \gamma b_{1} b_{2} \rho_{12}\right)$, the optimal reinsurance strategies of the big and small companies are the same as those in equation (29), while the optimal investment strategy of the big 
company is the same as that in equation (27) and the value function is given in equation (B.39).

(9) If $\left(\left(\lambda_{2} b_{1}-\rho_{12} \lambda_{1} b_{2}\right) /\left(b_{1} b_{2}^{2}\left(1-\rho_{12}^{2}\right)\right)\right)<-(\gamma / 2), e^{r_{0} T}$ $\geq-\left(2\left(\lambda_{2} b_{1}-\rho_{12} \lambda_{1} b_{2}\right) /\left(\gamma b_{1} b_{2}^{2}\left(1-\rho_{12}^{2}\right)\right)\right), \quad \rho_{12}<0$, $-\left(\lambda_{1} / \gamma b_{1} b_{2} \rho_{12}\right)>1$, and $e^{r_{0} T} \geq-\left(\lambda_{1} / \gamma b_{1} b_{2} \rho_{12}\right)$, the optimal reinsurance strategies of the big and small companies are

$$
\left(p_{1}^{*}(t), p_{2}^{*}(t)\right)= \begin{cases}(0,1), & 0 \leq t \leq t_{3}, \\ \left(\frac{\lambda_{1}}{\gamma b_{1}^{2}} \mathrm{e}^{-r_{0}(T-t)}+\frac{b_{2} \rho_{12}}{b_{1}}, 1\right), & t_{3}<t \leq t_{2}, \\ \left(\frac{\lambda_{1}}{\gamma b_{1}^{2}} \mathrm{e}^{-r_{0}(T-t)}, 0\right), & t_{2}<t \leq T .\end{cases}
$$

The optimal investment strategy of the big company is expressed as that in equation (11) and the value function is given by equation (B.42).

Proof. See Appendix B.

Remark 2. From Theorem 2 we conclude that the wealth has no influence on the optimal investment strategy. This can be explained by the risk tolerance of the exponential utility function. The risk tolerance is $-U_{x} / U_{x x}=1 / \gamma$, which is independent of the wealth. Thus, the optimal strategy is independent of the wealth. In addition, the optimal reinsurance strategy of the big company is not related to the wealth as well and that of the small company is either 1 or 0 , i.e., the small insurance company takes extreme or trivial strategy in this game.

Remark 3. In the case that the risky asset's price follows the GBM model, the optimal strategy of the big insurance company is

$$
\pi^{*}(t)=\frac{\left(\mu-r_{0}\right) \mathrm{e}^{-r_{0}(T-t)}}{\sigma^{2} \gamma} .
$$

Compared with equation (34), we see that the optimal investment strategy under the CEV model can be decomposed into two parts. One is

$$
M(t)=\frac{\left(\mu-r_{0}\right) e^{-r_{0}(T-t)}}{\sigma^{2} s^{2 \beta} \gamma},
$$

which is similar to the optimal strategy under the GBM model, but the volatility is stochastic. Thus, we call $M(t)$ as the moving GBM strategy. The other one is

$$
N(t)=1+\frac{\mu-r_{0}}{2 r_{0}}\left(1-e^{-2 r_{0} \beta(T-t)}\right),
$$

which reflects the insurance company's decision to hedge the volatility risk and we regard it as a correction factor.

The following corollary discusses the property of the correction factor.
Corollary 1. The correction factor $N(t)$ is a monotone decreasing function with respect to time $t$ and satisfies

$$
1 \leq N(t) \leq 1+\frac{\mu-r_{0}}{2 r_{0}}\left[1-e^{-2 r_{0} \beta T}\right]
$$

Proof. According to $\mu>r_{0}$ and $\beta>0$, we derive $N_{t}=-\beta\left(\mu-r_{0}\right) \mathrm{e}^{-2 r_{0} \beta(T-t)}<0$. It implies that the correction factor is a monotone decreasing function with respect to time $t$. Since

$$
N(0)=1+\frac{\mu-r_{0}}{2 r_{0}}\left[1-e^{-2 r_{0} \beta}\right]
$$

and $N(T)=1$, we obtain inequality (37).

Corollary 1 shows that the correction factor advises the big insurance company to invest more wealth in the risky asset at the beginning of the investment horizon and steadily decrease the amounts as time goes on.

Remark 4. We find that the optimal investment strategy is independent of the optimal reinsurance strategies. The main reason is that in this model, we assume that the financial market is not affected by the insurance market, which is used in a great deal of existing literature, such as Bai and Guo [3], $\mathrm{Gu}$ et al. [26], Gu et al. [29], and so on.

\section{Numerical Examples}

In this section, we provide some numerical simulations to illustrate our results. Because the optimal reinsurance strategy of the small company is either 1 or 0 , we analyze the optimal reinsurance and investment strategies of the big company here. Throughout numerical analysis, unless otherwise stated, the basic parameters are given by $a_{1}=1.5$, $a_{2}=0.5, b_{1}=3, b_{2}=1, \lambda_{1}=2, \lambda_{2}=1, r_{0}=0.3, \mu=0.5$, $\sigma=1, \beta=1, s=67, \rho_{12}= \pm 0.5, \gamma=0.5, T=10$, and $t=5$.

4.1. Numerical Simulations of the Big Company's Optimal Reinsurance Strategy. Figure 1 illustrates the influence of the risk averse coefficient $\gamma$ on the optimal reinsurance strategy $p_{1}^{*}(t)$. The relationship between $p_{1}^{*}(t)$ and $\gamma$ is negative. This can be attributed to the fact that a larger $\gamma$ means the big insurance company is a more risk-averse individual. With the increase of $\gamma$, the big company wants to purchase more reinsurance to avoid risk and undertake less risk itself.

Figure 2 shows the effect of $r_{0}$ on the optimal reinsurance strategy $p_{1}^{*}(t)$. As $r_{0}$ increases, the big insurance company will obtain more profit from investment in the risk-free asset. Therefore, it has more money to purchase the reinsurance and bear less risk itself, so $p_{1}^{*}(t)$ decreases with $r_{0}$.

Figure 3 indicates the impact of $\lambda_{1}$ on the optimal reinsurance strategy $p_{1}^{*}(t)$. We can see that a greater $\lambda_{1}$ yields a greater reinsurance strategy. This is because that as $\lambda_{1}$ increases, the cost of reinsurance will become more expensive and the big insurance company prefers to maintain a stable revenue by purchasing less reinsurance and undertake more by itself. 


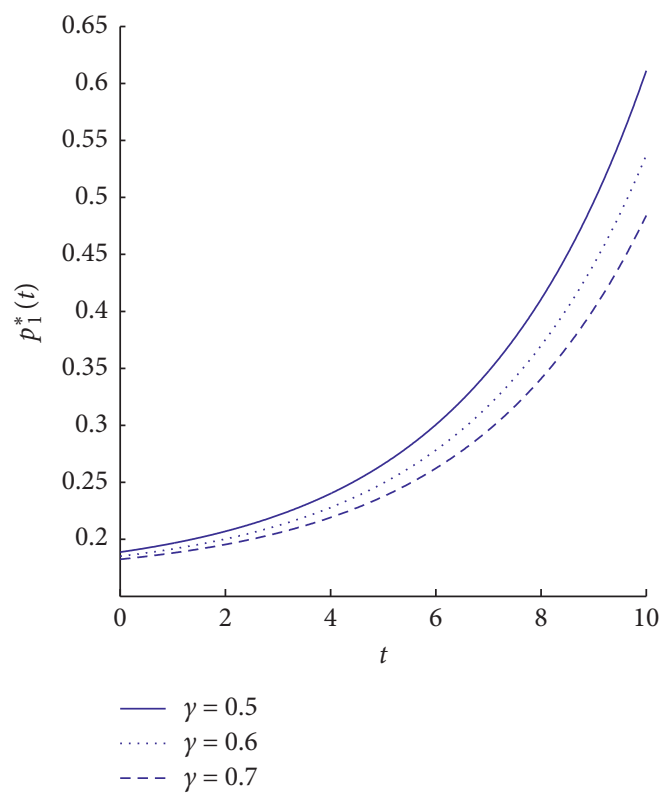

(a)

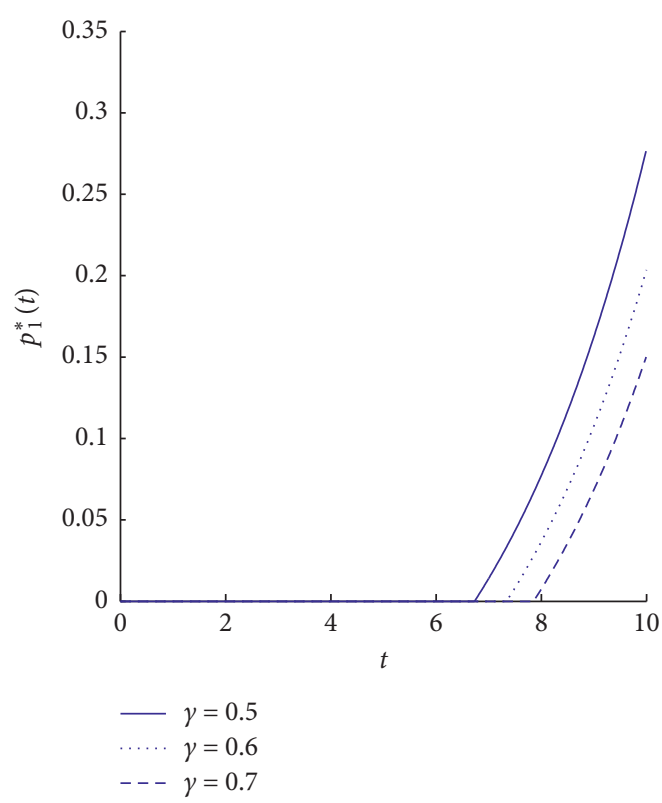

(b)

Figure 1: (a) The effect of $\gamma$ on the big company's optimal reinsurance strategy $p_{1}^{*}(t)$ when $\rho_{12}>0$. (b) The effect of $\gamma$ on the big company's optimal reinsurance strategy $p_{1}^{*}(t)$ when $\rho_{12}<0$.

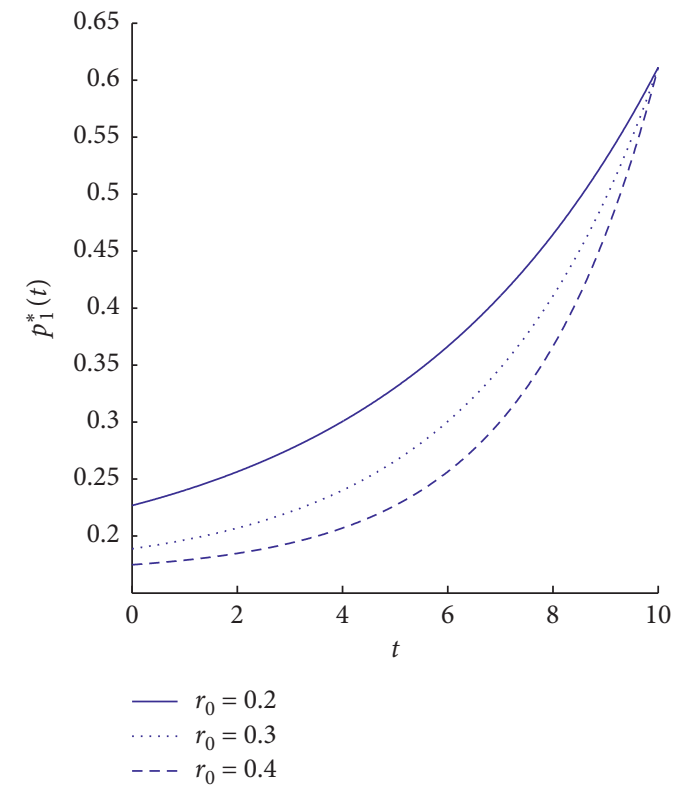

(a)

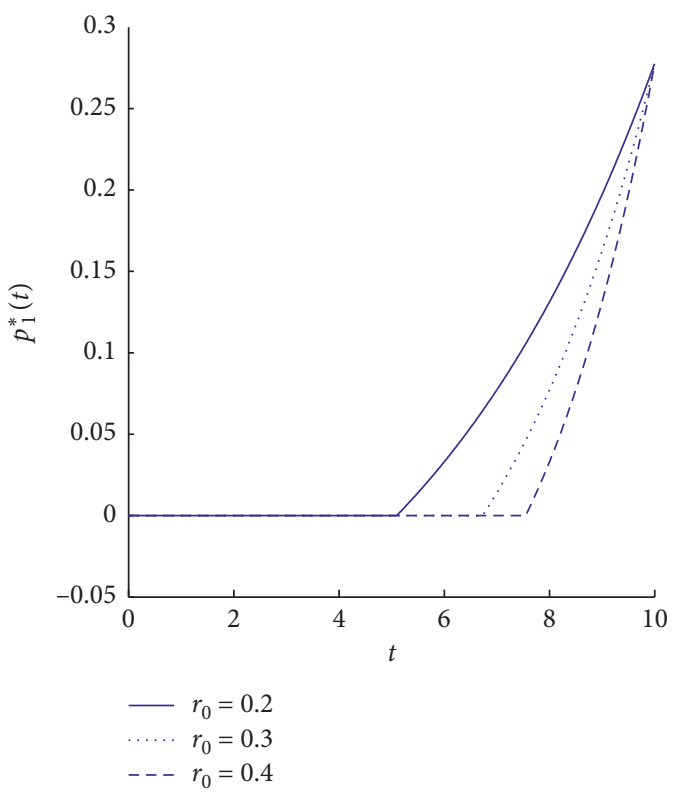

(b)

Figure 2: (a) The effect of $r_{0}$ on the big company's optimal reinsurance strategy $p_{1}^{*}(t)$ when $\rho_{12}>0$. (b) The effect of $r_{0}$ on the big company's optimal reinsurance strategy $p_{1}^{*}(t)$ when $\rho_{12}<0$.

As is shown in Figure 4, we find that $p_{1}^{*}(t)$ is a decreasing function of $b_{1}$. This can be explained by that $b_{1}$ implies the fluctuation of the big insurance company's claim process. When $b_{1}$ increases, the insurance company wants to purchase more reinsurance while undertaking less risk by itself.

From Figure 5, we can see if $\rho_{12}>0, b_{2}$ exerts a positive effect on the optimal reinsurance strategy $p_{1}^{*}(t)$ and if $\rho_{12}<0$, the effect is opposite. This can be attributed to that the fluctuations of the big and small companies' claim processes are more serious with $b_{2}$ rising when $\rho_{12}>0$. Therefore, the big company will take more risk, while when $\rho_{12}<0$, the fluctuation of the big insurance company's claim process is weaker with the volatility of the small company becoming stronger. So the big company will face less risk.

In Figure 6, we demonstrate the effect of the correlation coefficient between the big and small insurance companies' 


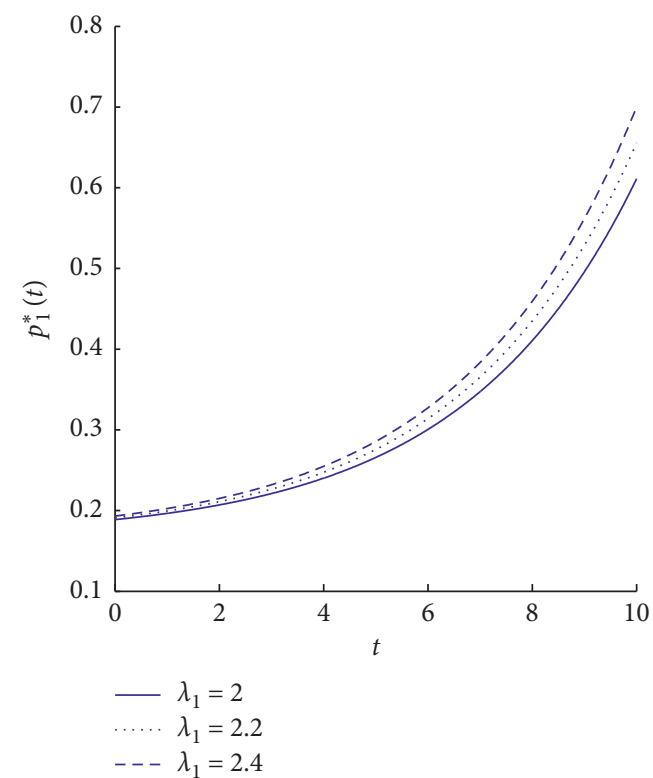

(a)

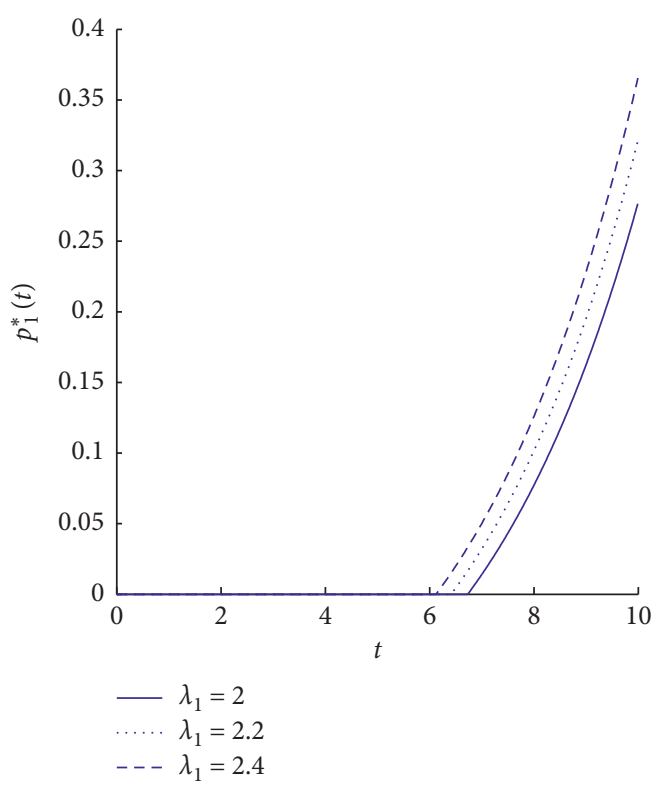

(b)

Figure 3: (a) The effect of $\lambda_{1}$ on the big company's optimal reinsurance strategy $p_{1}^{*}(t)$ when $\rho_{12}>0$. (b) The effect of $\lambda_{1}$ on the big company's optimal reinsurance strategy $p_{1}^{*}(t)$ when $\rho_{12}<0$.

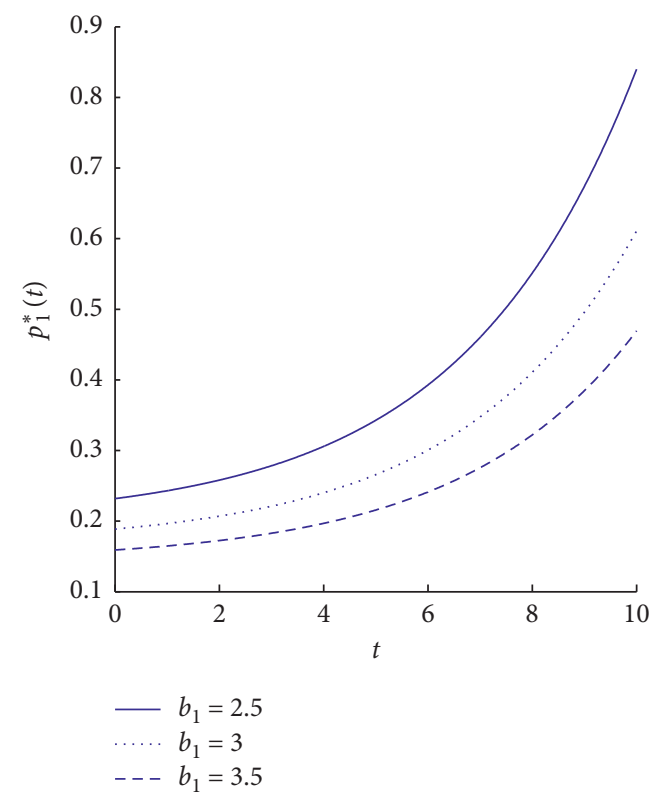

(a)

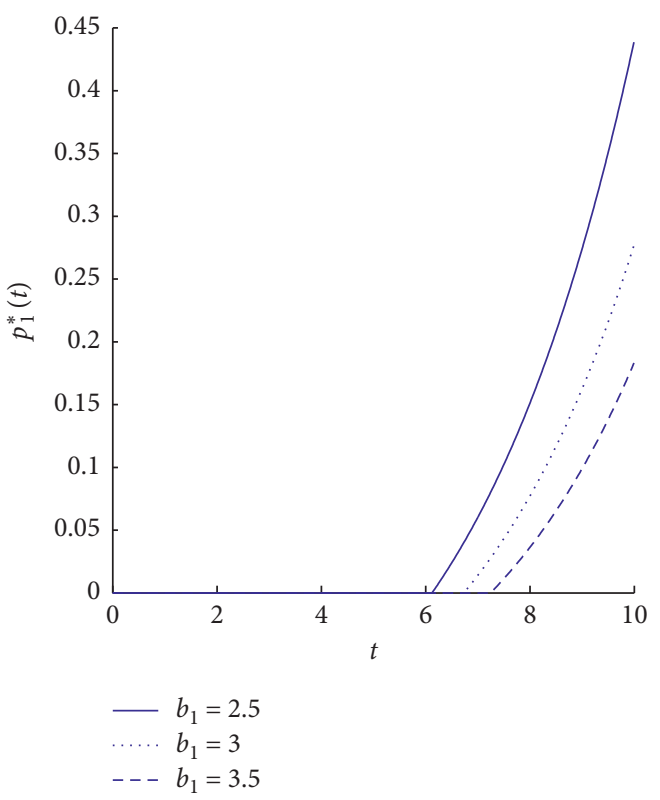

(b)

Figure 4: (a) The effect of $b_{1}$ on the big company's optimal reinsurance strategy $p_{1}^{*}(t)$ when $\rho_{12}>0$. (b) The effect of $b_{1}$ on the big company's optimal reinsurance strategy $p_{1}^{*}(t)$ when $\rho_{12}<0$.

risk processes $\rho_{12}$ on the optimal reinsurance strategy $p_{1}^{*}(t)$. We find that no matter $\rho_{12}$ is positive or negative, the higher $\rho_{12}$ is, the bigger $p_{1}^{*}(t)$ is. This is because that for the big company, the more relevant the relationship between two companies is, the greater the influence of the small company on the big company is. So the big company has to undertake more risk with $\rho_{12}$ rising.
4.2. Numerical Simulations of the Big Insurance Company's Optimal Investment Strategy. Figure 7(a) plots the evolution of the risky asset's price over time under the CEV model. According to the change trend of the risky asset's price, we plot the dynamic behaviors of the optimal investment strategy of the big insurance company in Figure $7(b)$ and we can see that the change trend of the 


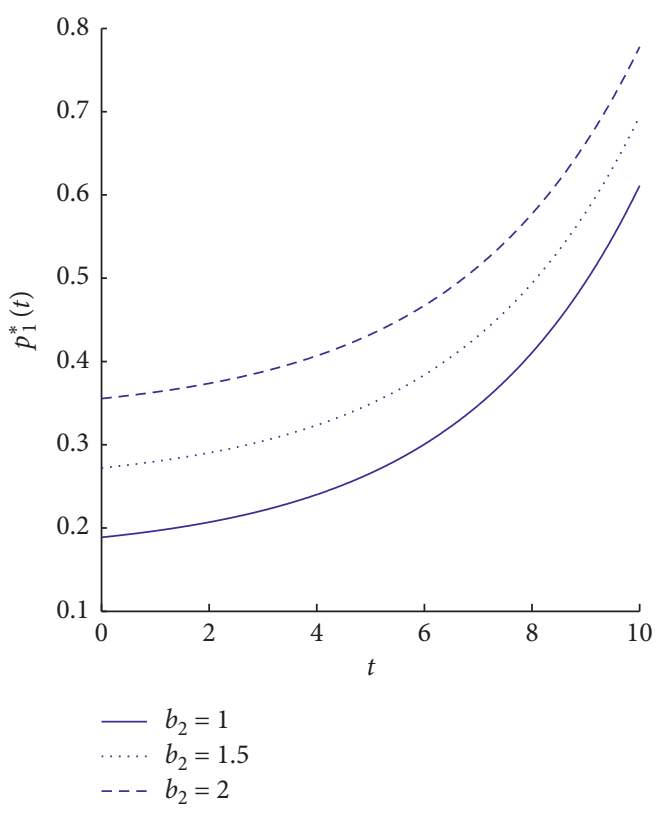

(a)

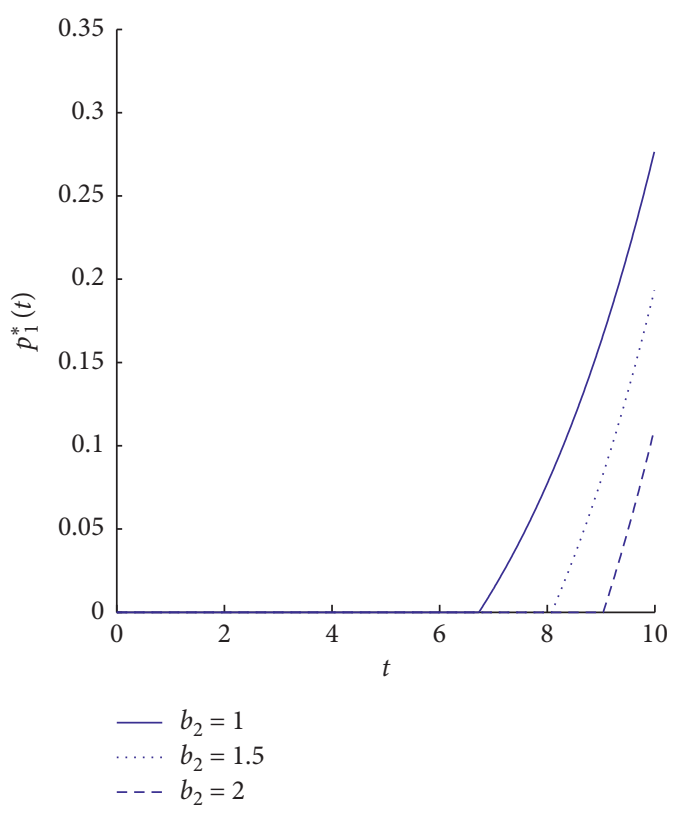

(b)

Figure 5: (a) The effect of $b_{2}$ on the big company's optimal reinsurance strategy $p_{1}^{*}(t)$ when $\rho_{12}>0$. (b) The effect of $b_{2}$ on the big company's optimal reinsurance strategy $p_{1}^{*}(t)$ when $\rho_{12}<0$.

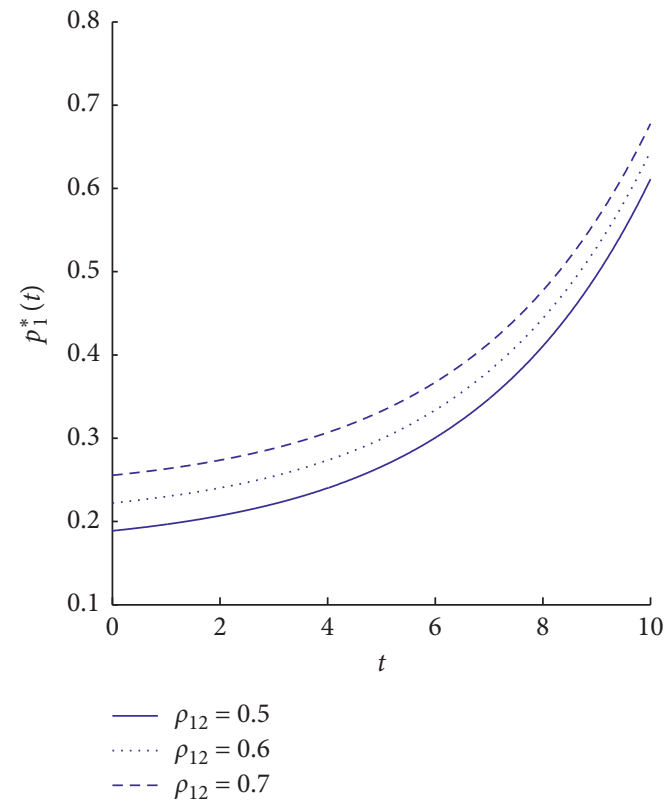

(a)

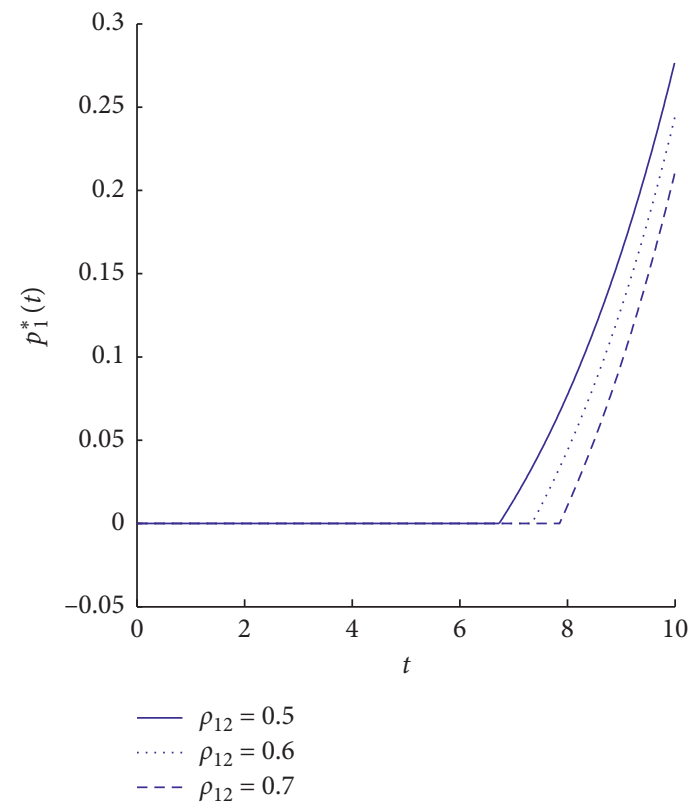

(b)

Figure 6: (a) The effect of $\rho_{12}$ on the big company's optimal reinsurance strategy $p_{1}^{*}(t)$ when $\rho_{12}>0$. (b) The effect of $\rho_{12}$ on the big company's optimal reinsurance strategy $p_{1}^{*}(t)$ when $\rho_{12}<0$.

optimal investment strategy is opposite to that of the risky asset's price, which can be attributed to the expression of the CEV model. When the price of risky asset is high, the big company should be cautious to invest, for the reason that the higher the risky asset's price is, the higher risk they will undertake.
In Figure 8(a), we find that the rate of the risky asset's return $\mu$ exerts positive effect on the optimal investment strategy $\pi^{*}(t)$. This is consistent with intuition. As $\mu$ increases, the big company will obtain more from investment. Therefore, it will increase the amounts invested in the risky asset. As shown in Figure 8(b), the optimal investment 


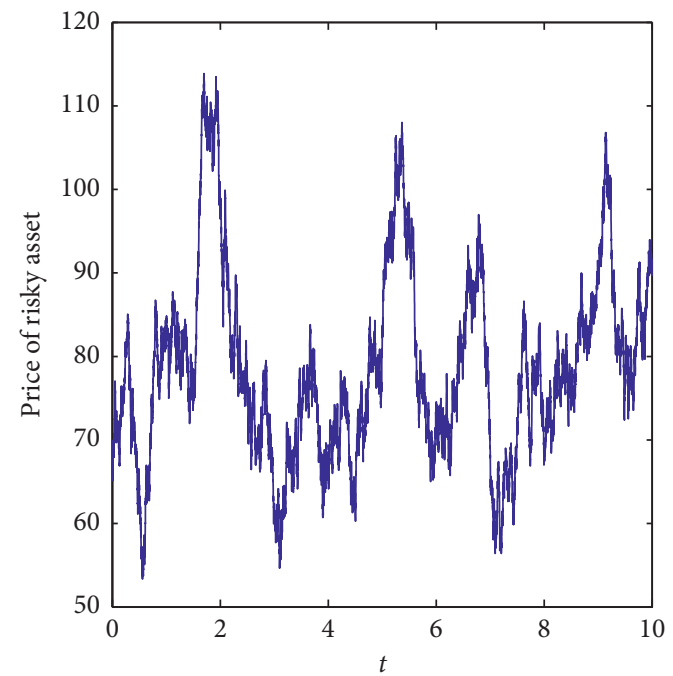

(a)

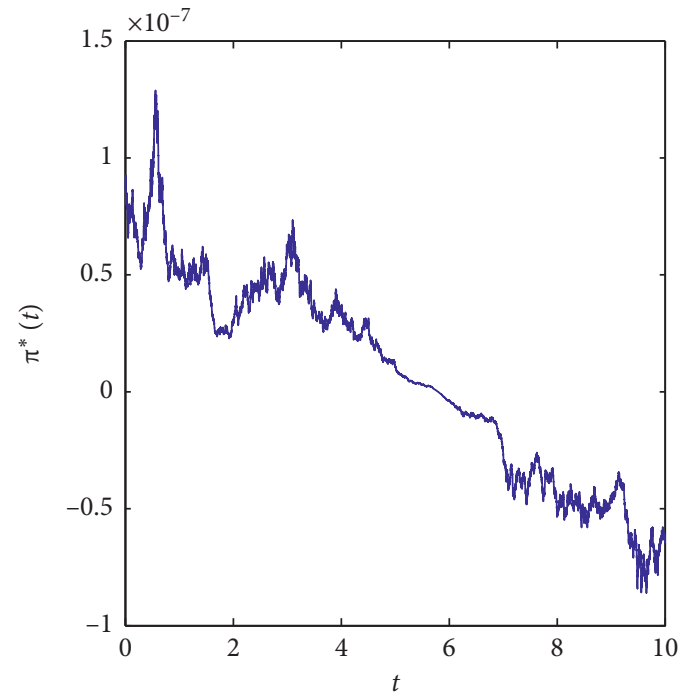

(b)

Figure 7: (a) Evolution of risky asset's price over time. (b) Evolution of optimal investment strategy of the insurer $\pi^{*}(t)$ over time.

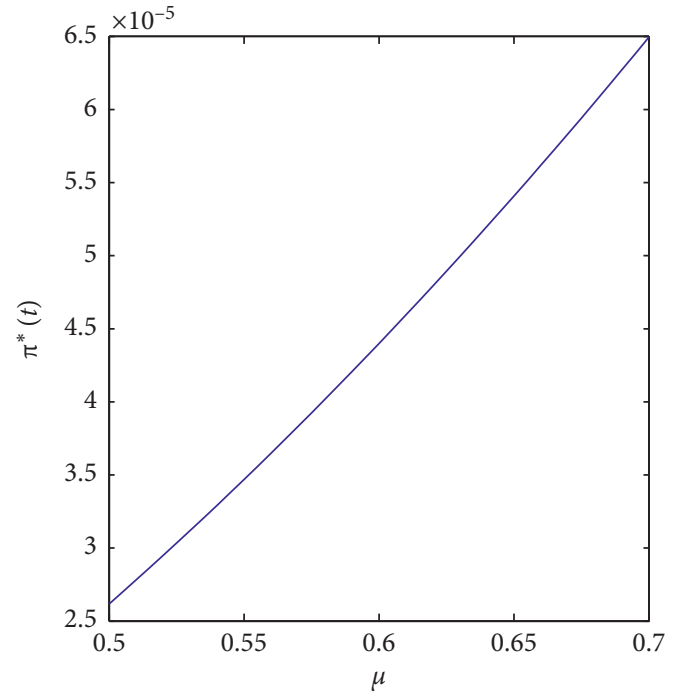

(a)

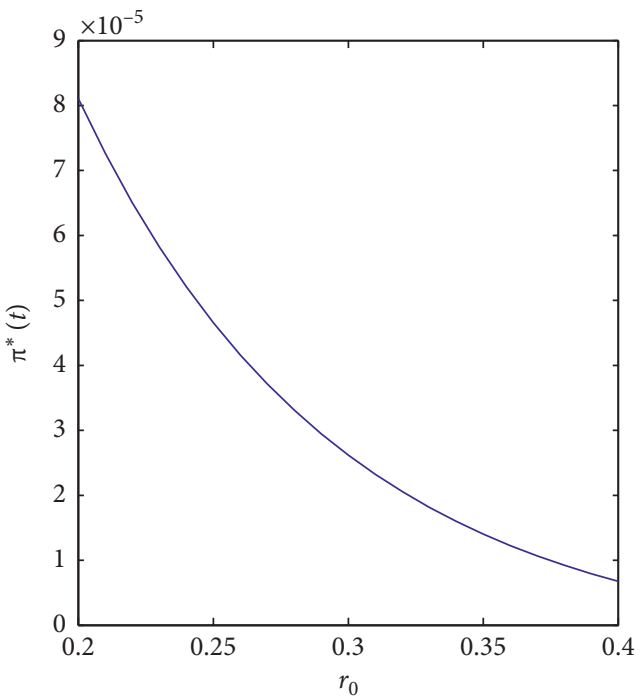

(b)

Figure 8: (a) The effect of $\mu$ on the optimal investment strategy of the big insurance company $\pi^{*}(t)$. (b) The effect of $r_{0}$ on the optimal investment strategy of the big insurance company $\pi^{*}(t)$.

strategy $\pi^{*}(t)$ is a decreasing function of the interest rate $r_{0}$. When the interest rate $r_{0}$ increases, the risk-free asset is more attractive. Then the big company will invest more in the risk-free asset and reduce investment in the risky asset.

Figure 9(a) indicates the impact of the risk averse coefficient $\gamma$ on the optimal investment strategy $\pi^{*}(t)$. We see that $\gamma$ exerts negative effect on $\pi^{*}(t)$, which means that the big company with the higher risk averse level will invest less in the risky asset to avoid risk. Figure 9(b) illustrates the effect of the elasticity coefficient $\beta$ on the optimal investment strategy $\pi^{*}(t)$. There is a negative relationship between $\pi^{*}(t)$ and $\beta$. This can be attributed to that a higher $\beta$ leads to a larger expected drop in volatility and increased probability of a large adverse movement in the risky asset'a price. Thus, 


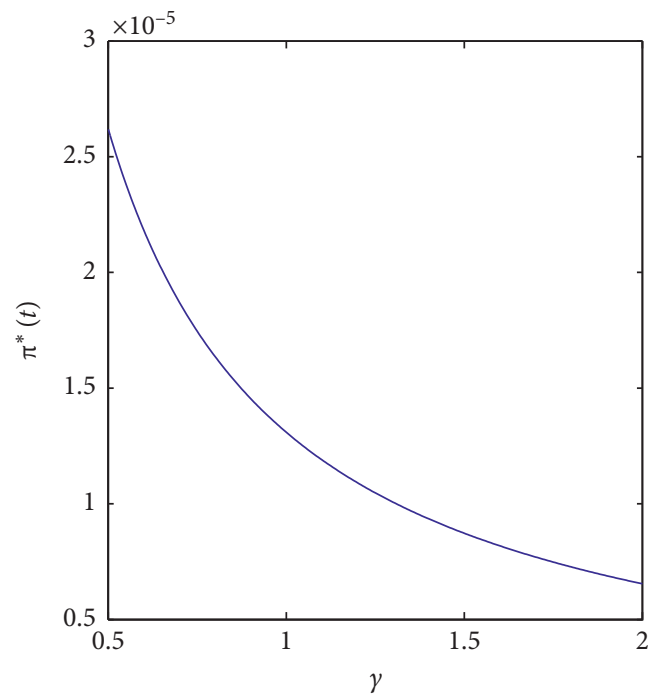

(a)

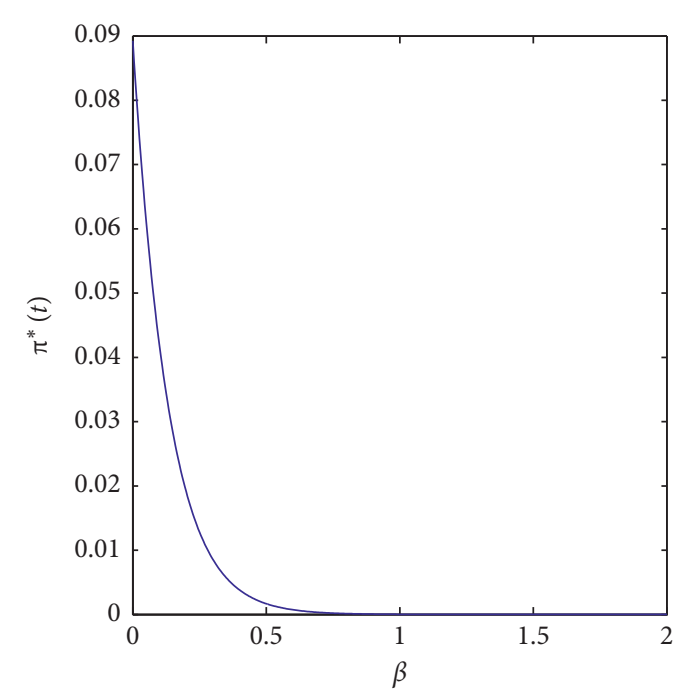

(b)

Figure 9: (a) The effect of $\gamma$ on the optimal investment strategy of the big insurance company $\pi^{*}(t)$. (b) The effect of $\beta$ on the optimal investment strategy of the big insurance company $\pi^{*}(t)$.

the big company will invest less in the risky asset as $\beta$ increases to reduce the risk from the investment in the risky asset.

\section{Conclusion}

This paper considers a stochastic differential game played between two insurance companies, a big one and a small one. In our model, the basic claim process is assumed to follow a Brownian motion with drift. Both of two insurance companies purchase the reinsurance, respectively. The big insurance company has more initial surplus than the small one, so the big company has sufficient asset to invest in the risky asset which is described by the CEV model and acquire new business like acting as a reinsurance company of other insurance companies, while the small company can only invest in the risk-free asset and purchase reinsurance. The game studied here is zero-sum where there is a single exponential utility. The big company is trying to maximize the expected exponential utility of the terminal wealth to keep its advantage on surplus while simultaneously the small company is trying to minimize the same quantity to reduce its disadvantage. Firstly, we describe the Nash equilibrium of the game and prove a verification theorem for the exponential utility. By solving the corresponding Fleming-Bellman-Isaacs equations, we derive the optimal reinsurance and investment strategies. Finally, numerical simulation is proposed to illustrate the impacts of the model parameters on the strategies. Through this paper, we find that (1) for such a game, the small insurance company takes extreme or trivial strategy, i.e., the optimal reinsurance strategy of the small company is either 1 or 0 ; (2) the optimal reinsurance and investment strategies of the big company are independent of the wealth process $X^{u_{1}, u_{2}}(t)$; (3) the effects of some model parameters on the big company's optimal reinsurance strategy is related to the correlation coefficient between the big and small companies' risk processes. In future work, we will consider more complex models, such as both two insurers can invest in the risky asset, or $n$ insurers will participate in the game.

\section{Appendix}

\section{A. Proof of Theorem 1}

Proof. By Itô's formula, we have

$$
\begin{aligned}
\mathrm{d} H^{u_{1}, u_{2}}\left(t, S(t), X^{u_{1}, u_{2}}(t)\right)= & \mathscr{A}^{u_{1}, u_{2}} H^{u_{1}, u_{2}}\left(t, S(t), X^{u_{1}, u_{2}}(t)\right) \mathrm{d} t \\
& +H_{x}^{u_{1}, u_{2}} \pi(t) \sigma S^{\beta}(t) \mathrm{d} W_{3}(t) \\
& +H_{x}^{u_{1}, u_{2}} p_{1}(t) b_{1} \mathrm{~d} W_{1}(t) \\
& -H_{x}^{u_{1}, u_{2}} p_{2}(t) b_{2} \mathrm{~d} W_{2}(t) \\
& +H_{s}^{u_{1}, u_{2}} \sigma S^{\beta+1}(t) \mathrm{d} W_{3}(t) .
\end{aligned}
$$

Take a sequence of bounded open sets $\mathrm{O}_{1}, \mathrm{O}_{2}, \mathrm{O}_{3}, \ldots$, with $O_{i} \subset O_{i+1} \subset O, \quad i=1,2, \ldots$, and $\cup_{i} O_{i}=O$. For $(s, x) \in O_{1}$, let $\tau_{i}$ be the exiting time of $(s, x)$ from $O_{i}$. Then $\tau_{i} \wedge T \longrightarrow T$ when $i \longrightarrow \infty$. Integrating from two sides of equation (A.1), we have 


$$
\begin{aligned}
& H^{u_{1}, u_{2}}\left(\tau_{i} \wedge T, S\left(\tau_{i} \wedge T\right), X^{u_{1}, u_{2}}\left(\tau_{i} \wedge T\right)\right) \\
& =H^{u_{1}, u_{2}}(0, s, x)+\int_{0}^{\tau_{i} \wedge T} \mathscr{A}^{u_{1}, u_{2}} H^{u_{1}, u_{2}}\left(\nu, S(\nu), X^{u_{1}, u_{2}}(\nu)\right) \mathrm{d} \nu+\int_{0}^{\tau_{i} \wedge T} H_{x}^{u_{1}, u_{2}} \pi(\nu) \sigma S^{\beta}(\nu) \mathrm{d} W_{3}(\nu) \\
& \quad+\int_{0}^{\tau_{i} \wedge T} H_{x}^{u_{1}, u_{2}} p_{1}(\nu) b_{1} \mathrm{~d} W_{1}(\nu)-\int_{0}^{\tau_{i} \wedge T} H_{x}^{u_{1}, u_{2}} p_{2}(\nu) b_{2} \mathrm{~d} W_{2}(\nu)+\int_{0}^{\tau_{i} \wedge T} \sigma S^{\beta+1}(\nu) \mathrm{d} W_{3}(\nu),
\end{aligned}
$$

then

$$
\begin{aligned}
& H^{u_{1}, u_{2}^{*}}\left(\tau_{i} \wedge T, S\left(\tau_{i} \wedge T\right), X^{u_{1}, u_{2}^{*}}\left(\tau_{i} \wedge T\right)\right) \\
& =H^{u_{1}, u_{2}^{*}}(0, s, x)+\int_{0}^{\tau_{i} \wedge T} \mathscr{A}^{u_{1}, u_{2}^{*}} H^{u_{1}, u_{2}^{*}}\left(\nu, S(\nu), X^{u_{1}, u_{2}^{*}}(\nu)\right) \mathrm{d} \nu+\int_{0}^{\tau_{i} \wedge T} H_{x}^{u_{1}, u_{2}^{*}} \pi(\nu) \sigma S^{\beta}(\nu) \mathrm{d} W_{3}(\nu) \\
& \quad+\int_{0}^{\tau_{i} \wedge T} H_{x}^{u_{1}, u_{2}^{*}} p_{1}(\nu) b_{1} \mathrm{~d} W_{1}(\nu)-\int_{0}^{\tau_{i} \wedge T} H_{x}^{u_{1}, u_{2}^{*}} p_{2}^{*}(\nu) b_{2} \mathrm{~d} W_{2}(\nu)+\int_{0}^{\tau_{i} \wedge T} H_{s}^{u_{1}, u_{2}^{*}} \sigma S^{\beta+1}(\nu) \mathrm{d} W_{3}(\nu) .
\end{aligned}
$$

Since the last five terms are square-integrable martingales with zero expectation, taking conditional expectation given $(t, s, x)$ on both sides of the above formula and taking equation (21) into account results that

$$
\begin{aligned}
& \mathrm{E}\left[H^{u_{1}, u_{2}^{*}}\left(\tau_{i} \wedge T, S\left(\tau_{i} \wedge T\right), X^{u_{1}, u_{2}^{*}}\left(\tau_{i} \wedge T\right)\right) \mid S(t)=s, X^{u_{1}, u_{2}^{*}}(t)=x\right] \\
& \quad=H^{u_{1}, u_{2}^{*}}(0, s, x)+\mathrm{E}\left[\int_{0}^{\tau_{i} \wedge T} \mathscr{A}^{u_{1}, u_{2}^{*}} H^{u_{1}, u_{2}^{*}}\left(\nu, S(\nu), X^{u_{1}, u_{2}^{*}}(\nu)\right) \mathrm{d} \nu \mid S(t)=s, X^{u_{1}, u_{2}^{*}}(t)=x\right] \\
& \quad \leq H^{u_{1}, u_{2}^{*}}(0, s, x) .
\end{aligned}
$$

By virtue of Lemma B.1, $H^{u_{1}, u_{2}^{*}}\left(\tau_{i} \wedge T, S\left(\tau_{i} \wedge T\right)\right.$, $\left.X^{u_{1}, u_{2}^{*}}\left(\tau_{i} \wedge T\right)\right), i=1,2, .$. are uniformly integrable. It is easy to see that the equality holds for $u_{1}=u_{1}^{*}$. Thus, we have

$$
\begin{aligned}
V^{u_{1}, u_{2}^{*}}(t, s, x) & =\mathrm{E}\left[U\left(X^{u_{1}, u_{2}^{*}}(T)\right) \mid S(t)=s, X^{u_{1}, u_{2}^{*}}(t)=x\right] \\
& =\lim _{i \longrightarrow \infty} \mathrm{E}\left[H^{u_{1}^{*}, u_{2}^{*}}\left(\tau_{i} \wedge T, S\left(\tau_{i} \wedge T\right), X^{u_{1}^{*}, u_{2}^{*}}\left(\tau_{i} \wedge T\right)\right) \mid S(t)=s, X^{u_{1}, u_{2}^{*}}(t)=x\right] \leq H^{u_{1}, u_{2}^{*}}(0, s, x) \\
& =H^{u_{1}^{*}, u_{2}^{*}}(0, s, x)=\lim _{i \longrightarrow \infty} \mathrm{E}\left[H^{u_{1}^{*}, u_{2}^{*}}\left(\tau_{i} \wedge T, S\left(\tau_{i} \wedge T\right), X^{u_{1}^{*}, u_{2}^{*}}\left(\tau_{i} \wedge T\right)\right) \mid S(t)=s, X^{u_{1}^{*}, u_{2}^{*}}(t)=x\right] \\
& =\mathrm{E}\left[U\left(X^{u_{1}^{*}, u_{2}^{*}}(T)\right) \mid S(t)=s, X^{u_{1}^{*}, u_{2}^{*}}(t)=x\right]=V^{u_{1}^{*}, u_{2}^{*}}(t, s, x),
\end{aligned}
$$

i.e., $V^{u_{1}, u_{2}^{*}}(t, s, x) \leq V^{u_{1}^{*}, u_{2}^{*}}(t, s, x)$. Similarly, we can prove

$$
V^{u_{1}^{*}, u_{2}^{*}}(t, s, x) \leq V^{u_{1}^{*}, u_{2}}(t, s, x) .
$$


Hence, $\left(u_{1}^{*}, u_{2}^{*}\right)$ is a saddle point for the game by Definition 1 and according to

$$
\begin{aligned}
& E\left[H^{u_{1}, u_{2}^{*}}\left(\tau_{i} \wedge T, S\left(\tau_{i} \wedge T\right), X^{u_{1}, u_{2}^{*}}\left(\tau_{i} \wedge T\right)\right) \mid S(t)=s, X^{u_{1}, u_{2}^{*}}(t)=x\right] \\
& \quad=H^{u_{1}, u_{2}^{*}}(t, s, x)+E\left[\int_{t}^{\tau_{i} \wedge T} \mathscr{A}^{u_{1}, u_{2}^{*}} H^{u_{1}, u_{2}^{*}}\left(\nu, S(\nu), X^{u_{1}, u_{2}^{*}}(\nu)\right) \mathrm{d} \nu \mid S(t)=s, X^{u_{1}, u_{2}^{*}}(t)=x\right] \\
& \quad \leq H^{u_{1}, u_{2}^{*}}(t, s, x),
\end{aligned}
$$

we can derive

$$
\begin{aligned}
V^{u_{1}, u_{2}^{*}}(t, s, x)= & \mathrm{E}\left[U\left(X^{u_{1}, u_{2}^{*}}(T)\right) \mid S(t)=s, X^{u_{1}, u_{2}^{*}}(t)=x\right] \\
= & \lim _{i \longrightarrow \infty} \mathrm{E}\left[H^{u_{1}, u_{2}^{*}}\left(\tau_{i} \wedge T, S\left(\tau_{i} \wedge T\right), X^{u_{1}, u_{2}^{*}}\left(\tau_{i} \wedge T\right)\right) \mid\right. \\
& \left.S(t)=s, X^{u_{1}, u_{2}^{*}}(t)=x\right] \leq H^{u_{1}, u_{2}^{*}}(t, s, x) .
\end{aligned}
$$

When $u_{1}=u_{1}^{*}$, the inequality in the above formula becomes an equality, i.e., $H(t, s, x)$ is the optimal value function.

\section{B. Proof of Theorem 2}

Proof. According to the exponential utility described by equation (9), we try to find the optimal value function in the following way:

$$
H(t, s, x)=-\frac{1}{\gamma} e^{-\gamma\left[e^{r_{0}(T-t)}(x-d(t))+g(t, s)\right]},
$$

with the boundary condition given by $g(T)=1$. Then

$$
\begin{aligned}
& H_{t}=-\gamma\left[-r_{0} \mathrm{e}^{r_{0}(T-t)}(x-d(t))-d_{t} \mathrm{e}^{r_{0}(T-t)}+g_{t}\right] H, H_{s}=-\gamma g_{s} H, H_{s s}=\left(\gamma^{2} g_{s}^{2}-\gamma g_{s s}\right) H, \\
& H_{x}=-\gamma \mathrm{e}^{r_{0}(T-t)} H, H_{x x}=\gamma^{2} \mathrm{e}^{2 r_{0}(T-t)} H, H_{s x}=\gamma^{2} \mathrm{e}^{r_{0}(T-t)} g_{s} H .
\end{aligned}
$$

From equation (B.2), we obtain $H_{x x}<0$, the infumum in equation $(21)$ is reached at $p_{2}^{*}(t)=0$ or $p_{2}^{*}(t)=1$. Let

$$
\widehat{p}_{2}(t)=\frac{\lambda_{2} b_{1}-\rho_{12} \lambda_{1} b_{2}}{b_{1} b_{2}^{2}\left(1-\rho_{12}^{2}\right)} \cdot \frac{H_{x}}{H_{x x}} \text {. }
$$

The first-order maximizing conditions for the optimal reinsurance and investment strategies of the big company give

$$
\begin{aligned}
& p_{1}^{*}(t)=-\frac{\lambda_{1} H_{x}}{b_{1}^{2} H_{x x}}+\frac{b_{2} \rho_{12} p_{2}^{*}(t)}{b_{1}}, \\
& \pi^{*}(t)=-\frac{\left(\mu-r_{0}\right) H_{x x}}{\sigma^{2} s^{2 \beta} H_{x x}}-\frac{s H_{x s}}{H_{x x}} .
\end{aligned}
$$

Substituting $\pi^{*}(t), p_{1}^{*}(t)$, and $p_{2}^{*}(t)$ into equations (20) and (21) yields

$$
\begin{aligned}
H_{t} & +r_{0} x H_{x}-\frac{\left(\mu-r_{0}\right)^{2} H_{x}^{2}}{2 \sigma^{2} s^{2} \beta H_{x x}}+D H_{x}+\frac{1}{2} p_{1}^{*}(t)^{2} b_{1}^{2} H_{x x}+\frac{1}{2} p_{2}^{*}(t)^{2} b_{2}^{2} H_{x x}+p_{1}^{*}(t) \lambda_{1} H_{x}-p_{2}^{*}(t) \lambda_{2} H_{x} \\
& -p_{1}^{*}(t) p_{2}^{*}(t) b_{1} b_{2} \rho_{12} H_{x x}+\mu s H_{s}+\frac{1}{2} \sigma^{2} s^{2 \beta+2} H_{s s}-\frac{s\left(\mu-r_{0}\right) H_{x} H_{x s}}{H_{x x}}-\frac{\sigma^{2} s^{2 \beta+2} H_{x s}^{2}}{2 H_{x x}}=0 .
\end{aligned}
$$

Inserting derivatives in equation (B.2) into equation (B.5), we obtain

$$
\begin{gathered}
g_{t}+r_{0} s g_{s}+\frac{1}{2} \sigma^{2} s^{2 \beta+2} g_{s s}+\frac{\left(\mu-r_{0}\right)^{2}}{2 \gamma \sigma^{2} s^{2 \beta}}+r_{0} e^{r_{0}(T-t)} d(t)-d_{t} e^{r_{0}(T-t)}+D e^{r_{0}(T-t)}+p_{1}^{*}(t) \lambda_{1} e^{r_{0}(T-t)} \\
-p_{2}^{*}(t) \lambda_{2} e^{r_{0}(T-t)}-\frac{1}{2} p_{1}^{*}(t)^{2} b_{1}^{2} \gamma e^{2 r_{0}(T-t)}-\frac{1}{2} p_{2}^{*}(t)^{2} b_{2}^{2} \gamma e^{2 r_{0}(T-t)}+p_{1}^{*}(t) p_{2}^{*}(t) b_{1} b_{2} \gamma e^{2 r_{0}(T-t)}=0 .
\end{gathered}
$$

In the following section, we try to find the solutions to equations (20) and (21) in the following cases.
Case B.1. $\left(\left(\lambda_{2} b_{1}-\rho_{12} \lambda_{1} b_{2}\right) /\left(b_{1} b_{2}^{2}\left(1-\rho_{12}^{2}\right)\right)\right) \geq-(\gamma / 2)$. 
If $\left(\left(\lambda_{2} b_{1}-\rho_{12} \lambda_{1} b_{2}\right) /\left(b_{1} b_{2}^{2}\left(1-\rho_{12}^{2}\right)\right)\right) \geq-(\gamma / 2), \quad$ then $\widehat{p}_{2}(t)<(1 / 2)$. We have the optimal reinsurance strategies of the small and big company $p_{2}^{*}(t)=1$ and

$$
\widehat{p}_{1}(t)=\frac{\lambda_{1}}{\gamma b_{1}^{2}} e^{-r_{0}(T-t)}+\frac{b_{2} \rho_{12}}{b_{1}} \text {. }
$$

Equation (B.7) shows that $\widehat{p}_{1}(t) \in(0, \infty)$ is equivalent to

$$
t \geq t_{1}=T-\frac{1}{r_{0}} \ln \left(-\frac{\lambda_{1}}{\gamma b_{1} b_{2} \rho_{12}}\right) .
$$

(1) If $\rho_{12} \geq 0$, we derive the optimal reinsurance strategies of the big and small companies

$$
\left(p_{1}^{*}(t), p_{2}^{*}(t)\right)=\left(\frac{\lambda_{1}}{\gamma b_{1}^{2}} \mathrm{e}^{-r_{0}(T-t)}+\frac{b_{2} \rho_{12}}{b_{1}}, 1\right), \quad 0 \leq t \leq T .
$$

Inputting equation (B.9) into equation (B.6) implies

$$
\begin{aligned}
& e^{r_{0}(T-t)}\left\{r_{0} d(t)-d(t)+D-\lambda_{2}+\frac{\lambda_{1} b_{2} \rho_{12}}{b_{1}}-\frac{1}{2} b_{2}^{2} \gamma\left(1-\rho_{12}^{2}\right) e^{r_{0}(T-t)}\right\} \\
& +g_{t}+r_{0} s g_{s}+\frac{1}{2} \sigma^{2} s^{2 \beta+2} g_{s s}+\frac{\left(\mu-r_{0}\right)^{2}}{2 \gamma \sigma^{2} s^{2} \beta}+\frac{\lambda_{1}^{2}}{2 \gamma b_{1}^{2}}=0 .
\end{aligned}
$$

Equation (B.10) can be decomposed into two equations by separating variables

$$
\begin{gathered}
d_{t}-r_{0} d(t)-D+\lambda_{2}-\frac{\lambda_{1} b_{2} \rho_{12}}{b_{1}}+\frac{1}{2} b_{2}^{2} \gamma\left(1-\rho_{12}^{2}\right) \mathrm{e}^{r_{0}(T-t)}=0, \\
g_{t}+r_{0} s g_{s}+\frac{1}{2} \sigma^{2} s^{2 \beta+2} g_{s s}+\frac{\left(\mu-r_{0}\right)^{2}}{2 \gamma \sigma^{2} s^{2 \beta}}+\frac{\lambda_{1}^{2}}{2 \gamma b_{1}^{2}}=0 .
\end{gathered}
$$

Taking the boundary condition $d(T)=0$ into account, the solution to equation (B.11) is

$$
\begin{aligned}
d(t)= & -\frac{1}{r_{0}}\left(D+\frac{\lambda_{1} b_{2} \rho_{12}}{b_{1}}-\lambda_{2}\right)\left(1-e^{-r_{0}(T-t)}\right) \\
& +\frac{\gamma b_{2}^{2}\left(1-\rho_{12}^{2}\right)}{4 r_{0}}\left(e^{r_{0}(T-t)}-e^{-r_{0}(T-t)}\right) .
\end{aligned}
$$

In order to solve equation (B.12), we define

$$
g(t, s)=m(t, v), \quad v=s^{-2 \beta},
$$

and the boundary condition is $m(T, v)=0$. Then

$$
\begin{aligned}
& g_{t}=m_{t}, \\
& g_{s}=-2 \beta s^{-2 \beta-1} m_{v}, \\
& g_{s s}=2 \beta(2 \beta+1) s^{-2 \beta-2} m_{v}+4 \beta^{2} s^{-4 \beta-2} m_{v v} .
\end{aligned}
$$

Substituting these derivatives into equation (B.15) yields

$$
\begin{gathered}
m_{t}-2 r_{0} \beta v m_{v}+\beta(2 \beta+1) \sigma^{2} m_{v}+2 \beta^{2} \sigma^{2} v m_{v v} \\
+\frac{\left(\mu-r_{0}\right)^{2} v}{2 \sigma^{2} \gamma}+\frac{\lambda_{1}^{2}}{2 \gamma b_{1}^{2}}=0 .
\end{gathered}
$$

We try to find a solution to equation (B.16) with the following structure:

$$
m(t, v)=A(t)+B(t) v,
$$

and the boundary conditions are $A(T)=0$ and $B(T)=0$. Plugging equation (B.17) into equation (B.15), we derive

$$
\begin{aligned}
A_{t} & +\beta(2 \beta+1) \sigma^{2} B(t)+\frac{\lambda_{1}^{2}}{2 \gamma b_{1}^{2}} \\
& +v\left\{B_{t}-2 r_{0} \beta B(t)+\frac{\left(\mu-r_{0}\right)^{2}}{2 \sigma^{2} \gamma}\right\}=0 .
\end{aligned}
$$

By matching coefficients, $A(t)$ and $B(t)$ satisfy the following equations:

$$
\begin{gathered}
A_{t}+\beta(2 \beta+1) \sigma^{2} B(t)+\frac{\lambda_{1}^{2}}{2 \gamma b_{1}^{2}}=0, \\
B_{t}-2 r_{0} \beta B(t)+\frac{\left(\mu-r_{0}\right)^{2}}{2 \sigma^{2} \gamma}=0 .
\end{gathered}
$$

Considering the boundary conditions $A(T)=0$ and $B(T)=0$, the solutions to equation (B.19) are

$$
\begin{aligned}
& A(t)=\frac{\lambda_{1}^{2}}{2 \gamma b_{1}^{2}}(T-t)+\beta(2 \beta+1) \sigma^{2} \int_{t}^{T} B(\nu) \mathrm{d} \nu, \\
& B(t)=\frac{\left(\mu-r_{0}\right)^{2}}{4 \sigma^{2} \gamma r_{0} \beta}\left(1-\mathrm{e}^{-2 r_{0} \beta(T-t)}\right) .
\end{aligned}
$$

Thus, we obtain 


$$
\left.H(t, s, x)=-\frac{1}{\gamma} e^{-\gamma\left[e^{r}(T-t)\right.}\left(x-d_{1}(t)\right)+A_{1}(t)+B(t) s^{-2 \beta}\right],
$$

where

$$
\begin{aligned}
d_{1}(t)= & -\frac{1}{r_{0}}\left(D+\frac{\lambda_{1} b_{2} \rho_{12}}{b_{1}}-\lambda_{2}\right)\left(1-e^{-r_{0}(T-t)}\right) \\
& +\frac{\gamma b_{2}^{2}\left(1-\rho_{12}^{2}\right)}{4 r_{0}}\left(e^{r_{0}(T-t)}-e^{-r_{0}(T-t)}\right), \\
A_{1}(t)= & \frac{\lambda_{1}^{2}}{2 \gamma b_{1}^{2}}(T-t)+\beta(2 \beta+1) \sigma^{2} \int_{t}^{T} B(\nu) \mathrm{d} \nu,
\end{aligned}
$$

and $B(t)$ is given by equation (B.20).

(2) If $\rho_{12}<0$ and $-\left(\lambda_{1} / \gamma b_{1} b_{2} \rho_{12}\right) \leq 1$, we have the optimal reinsurance strategies of the big and small companies

$$
\left(p_{1}^{*}(t), p_{2}^{*}(t)\right)=(0,1), \quad 0 \leq t \leq T .
$$

Putting equation (B.23) into equation (B.6), we derive

$$
\begin{gathered}
\mathrm{e}^{\mathrm{r}_{0}(\mathrm{~T}-\mathrm{t})}\left\{r_{0} d(t)-d_{t}+D-\lambda_{2}-\frac{1}{2} b_{2}^{2} \gamma \mathrm{e}^{r_{0}(T-t)}\right\}+g_{t} \\
+r_{0} s g_{s}+\frac{1}{2} \sigma^{2} s^{2 \beta+2} g_{s s}+\frac{\left(\mu-r_{0}\right)^{2}}{2 \gamma \sigma^{2} s^{2 \beta}}=0 .
\end{gathered}
$$

We can solve equation (B.24) like Case B.1 (1) and get

$$
H(t, s, x)=-\frac{1}{\gamma} e^{-\gamma\left[e^{r_{0}(T-t)}\left(x-d_{2}(t)\right)+A_{2}\left((t)+B(t) s^{-2 \beta}\right]\right.},
$$

where

$$
\begin{aligned}
& d_{2}(t)=-\frac{1}{r_{0}}\left(D-\lambda_{2}\right)\left(1-e^{-r_{0}(T-t)}\right)+\frac{\gamma b_{2}^{2}}{4 r_{0}}\left(e^{r_{0}(T-t)}-e^{-r_{0}(T-t)}\right), \\
& A_{2}(t)=\beta(2 \beta+1) \sigma^{2} \int_{t}^{T} B(\nu) \mathrm{d} \nu,
\end{aligned}
$$

with $B(t)$ given in equation (B.20).

(3) If $\rho_{12}<0,-\left(\lambda_{1} / \gamma b_{1} b_{2} \rho_{12}\right)>1$ and $e^{r_{0} T}<-\quad\left(\lambda_{1} / \gamma\right.$ $\left.b_{1} b_{2} \rho_{12}\right)$, the optimal reinsurance strategies of the big and small companies are the same as those in equation (B.9) and the expression of $H(t, s, x)$ is given by equation (B.21).

(4) If $\rho_{12}<0,-\left(\lambda_{1} / \gamma b_{1} b_{2} \rho_{12}\right)>1$ and $e^{r_{0} T} \geq-\left(\lambda_{1} / \gamma b_{1}\right.$ $\left.b_{2} \rho_{12}\right)$, when $0 \leq t \leq t_{1}$, the optimal reinsurance strategies of the big and small companies are expressed as those in equation (B.23) and when $t_{1}<t \leq T, p_{1}^{*}(t)$ and $p_{2}^{*}(t)$ are the same as those in equation (B.9), i.e.,

$$
\left(p_{1}^{*}(t), p_{2}^{*}(t)\right)= \begin{cases}(0,1), & 0 \leq t \leq t_{1}, \\ \left(\frac{\lambda_{1}}{\gamma b_{1}^{2}} e^{-r_{0}(T-t)}+\frac{b_{2} \rho_{12}}{b_{1}}, 1\right), & t_{1}<t \leq T .\end{cases}
$$

By similar derivatives, noting that $H(t, s, x)$ is continuous at $t=t_{1}$ and taking the boundary conditions into account, we obtain

$$
H(t, s, x)= \begin{cases}-\frac{1}{\gamma} e^{-\gamma\left[e^{r_{0}(T-t)}\left(x-d_{3}(t)\right)+A_{3}(t)+B(t) s^{-2 \beta}\right]}, & 0 \leq t \leq t_{1}, \\ -\frac{-}{\gamma} e^{-\gamma\left[e^{r_{0}(T-t)}\left(x-d_{1}(t)\right)+A_{1}(t)+B(t) s^{-2 \beta}\right]}, & t_{1}<t \leq T,\end{cases}
$$

where

$$
\begin{aligned}
d_{3}(t)= & -\frac{1}{r_{0}}\left(D-\lambda_{2}\right)\left(1-e^{-r_{0}\left(t_{1}-t\right)}\right)-\frac{\lambda_{1} b_{2} \rho_{12}}{r_{0} b_{1}} \\
& \cdot\left(e^{-r_{0}\left(t_{1}-t\right)}-e^{-r_{0}(T-t)}\right)+\frac{\gamma b_{2}^{2}}{4 r_{0}}\left(e^{r_{0}(T-t)}-e^{-r_{0}(T-t)}\right) \\
& -\frac{\gamma b_{2}^{2} \rho_{12}^{2}}{4 r_{0}}\left(\mathrm{e}^{r_{0}\left(T+t-2 t_{1}\right)}-\mathrm{e}^{-r_{0}(T-t)}\right), \\
A_{3}(t)= & \frac{\lambda_{1}^{2}}{2 \gamma b_{1}^{2}}\left(T-t_{1}\right)+\beta(2 \beta+1) \sigma^{2} \int_{t}^{T} B(\nu) \mathrm{d} \nu,
\end{aligned}
$$

and $B(t), d_{1}(t)$ and $A_{1}(t)$ are given in equations (B.20) and (B.21).

Case B.2. $\left(\left(\lambda_{2} b_{1}-\rho_{12} \lambda_{1} b_{2}\right) /\left(b_{1} b_{2}^{2}\left(1-\rho_{12}^{2}\right)\right)\right)<-(\gamma / 2)$ and $e^{r_{0} T}<-\left(2\left(\lambda_{2} b_{1}-\rho_{12} \lambda_{1} b_{2}\right) / \gamma b_{1} b_{2}^{2}\left(1-\rho_{12}^{2}\right)\right)$.

If $\quad\left(\left(\lambda_{2} b_{1}-\rho_{12} \lambda_{1} b_{2}\right) /\left(b_{1} b_{2}^{2}\left(1-\rho_{12}^{2}\right)\right)\right)<-(\gamma / 2)$ and $\mathrm{e}^{r_{0} T}<-\left(2\left(\lambda_{2} b_{1}-\rho_{12} \lambda_{1} b_{2}\right) / \gamma b_{1} b_{2}^{2}\left(1-\rho_{12}^{2}\right)\right)$, then $\hat{p}_{2}(t) \geq$ $1 / 2$. The optimal reinsurance strategies of the big and small companies are as following:

$$
\left(p_{1}^{*}(t), p_{2}^{*}(t)\right)=\left(\frac{\lambda_{1}}{\gamma b_{1}^{2}} e^{-r_{0}(T-t)}, 0\right), \quad 0 \leq t \leq T
$$

and equation (B.6) is simplified into

$$
\begin{gathered}
e^{r_{0}(T-t)}\left\{r_{0} d(t)-d_{t}+D\right\}+g_{t}+r_{0} s g_{s} \\
+\frac{1}{2} \sigma^{2} s^{2 \beta+2} g_{s s}+\frac{\left(\mu-r_{0}\right)^{2}}{2 \gamma \sigma^{2} s^{2 \beta}}+\frac{\lambda_{1}^{2}}{2 \gamma b_{1}^{2}}=0 .
\end{gathered}
$$

Thus, $H(t, s, x)$ satisfies

$$
H(t, s, x)=-\frac{1}{\gamma} e^{-\gamma\left[e^{r_{0}(T-t)}\left(x-d_{4}(t)\right)+A_{4}(t)+B(t) s^{-2 \beta}\right]},
$$

where 


$$
\begin{aligned}
& d_{4}(t)=-\frac{D}{r_{0}}\left(1-e^{-r_{0}(T-t)}\right), \\
& A_{4}(t)=\frac{\lambda_{1}^{2}}{2 \gamma b_{1}^{2}}(T-t)+\beta(2 \beta+1) \sigma^{2} \int_{t}^{T} B(\nu) \mathrm{d} \nu,
\end{aligned}
$$

with $B(t)$ given by equation (B.20).

Case B.3. $\quad\left(\left(\lambda_{2} b_{1}-\rho_{12} \lambda_{1} b_{2}\right) /\left(b_{1} b_{2}^{2}\left(1-\rho_{12}^{2}\right)\right)\right)<-(\gamma / 2)$ and $e^{r_{0} T} \geq-\left(2\left(\lambda_{2} b_{1}-\rho_{12} \lambda_{1} b_{2}\right) / \gamma b_{1} b_{2}^{2}\left(1-\rho_{12}^{2}\right)\right)$.

If $\quad\left(\left(\lambda_{2} b_{1}-\rho_{12} \lambda_{1} b_{2}\right) /\left(b_{1} b_{2}^{2}\left(1-\rho_{12}^{2}\right)\right)\right)<-(\gamma / 2) \quad$ and $e^{r_{0} T} \geq-\left(2\left(\lambda_{2} b_{1}-\rho_{12} \lambda_{1} b_{2}\right) / \gamma b_{1} b_{2}^{2}\left(1-\rho_{12}^{2}\right)\right)$, we derive the optimal reinsurance strategy of the small company

$$
p_{2}^{*}(t)= \begin{cases}1, & 0 \leq t \leq t_{2} \\ 0, & t_{2}<t \leq T\end{cases}
$$

where $\quad t_{2}=T-\left(1 / r_{0}\right) \ln \left(-\left(2\left(\lambda_{2} b_{1}-\rho_{12} \lambda_{1} b_{2}\right) / \gamma b_{1} b_{2}^{2}(1-\right.\right.$ $\left.\left.\rho_{12}^{2}\right)\right)$ ). Let $t_{3}=\min \left(t_{1}, t_{2}\right)$.

(1) If $\rho_{12} \geq 0$, when $0 \leq t \leq t_{2}$, the optimal reinsurance strategies of the big and small companies are expressed as those in equation (B.9) and when $t_{2}<t \leq T, p_{1}^{*}(t)$ and $p_{2}^{*}(t)$ are the same as those in equation (B.30), i.e.,

$$
\left(p_{1}^{*}(t), p_{2}^{*}(t)\right)= \begin{cases}\left(\frac{\lambda_{1}}{\gamma b_{1}^{2}} e^{-r_{0}(T-t)}+\frac{b_{2} \rho_{12}}{b_{1}}, 1\right), & 0 \leq t \leq t_{2}, \\ \left(\frac{\lambda_{1}}{\gamma b_{1}^{2}} e^{-r_{0}(T-t)}, 0\right), & t_{2}<t \leq T .\end{cases}
$$

Similarly, the expression of $H(t, s, x)$ is

$$
H(t, s, x)= \begin{cases}-\frac{1}{\gamma} e^{-\gamma\left[e^{r_{0}(T-t)}\left(x-d_{5}(t)\right)+A_{5}(t)+B(t) s^{-2 \beta}\right]}, & 0 \leq t \leq t_{2}, \\ -\frac{1}{\gamma} e^{-\gamma\left[e^{r_{0}(T-t)}\left(x-d_{4}(t)\right)+A_{4}(t)+B(t) s^{-2 \beta}\right]}, & t_{2}<t \leq T,\end{cases}
$$

where

$$
\begin{aligned}
d_{5}(t)= & -\frac{D}{r_{0}}\left(1-e^{-r_{0}(T-t)}\right)-\frac{\lambda_{1} b_{2} \rho_{12}-\lambda_{2} b_{1}}{r_{0} b_{1}}\left(1-e^{-r_{0}\left(t_{2}-t\right)}\right) \\
& +\frac{\gamma b_{2}^{2}\left(1-\rho_{12}^{2}\right)}{4 r_{0}}\left(e^{r_{0}(T-t)}-e^{r_{0}\left(T+t-2 t_{2}\right)}\right), \\
A_{5}(t)= & \frac{\lambda_{1}^{2}}{2 \gamma b_{1}^{2}}(T-t)+\beta(2 \beta+1) \sigma^{2} \int_{t}^{T} B(\nu) \mathrm{d} \nu,
\end{aligned}
$$

and $B(t), d_{4}(t), A_{4}(t)$ are given in equations (B.20) and (B.33).

(2) If $\rho_{12}<0$ and $-\left(\lambda_{1} / \gamma b_{1} b_{2} \rho_{12}\right) \leq 1$, when $0 \leq t \leq t_{2}$, the optimal reinsurance strategies of the big and small companies are expressed as those in equation (B.23) and when $t_{2}<t \leq T, p_{1}^{*}(t)$ and $p_{2}^{*}(t)$ are the same as those in equation (B.30), i.e.,

$\left(p_{1}^{*}(t), p_{2}^{*}(t)\right)= \begin{cases}(0,1), & 0 \leq t \leq t_{2}, \\ \left(\frac{\lambda_{1}}{\gamma b_{1}^{2}} e^{-r_{0}(T-t)}, 0\right), & t_{2}<t \leq T,\end{cases}$

while $H(t, s, x)$ is

$$
H(t, s, x)= \begin{cases}-\frac{1}{\gamma} e^{-\gamma\left[e^{r_{0}(T-t)}\left(x-d_{6}(t)\right)+A_{6}(t)+B(t) s^{-2 \beta}\right]}, & 0 \leq t \leq t_{2}, \\ & \\ -\frac{1}{\gamma} e^{-\gamma\left[e^{r_{0}(T-t)}\left(x-d_{4}(t)\right)+A_{4}(t)+B(t) s^{-2 \beta}\right]}, & t_{2}<t \leq T,\end{cases}
$$

where

$$
\begin{aligned}
d_{6}(t)= & -\frac{D}{r_{0}}\left(1-e^{-r_{0}(T-t)}\right)+\frac{\lambda_{2}}{r_{0}}\left(1-e^{-r_{0}\left(t_{2}-t\right)}\right) \\
& +\frac{\gamma b_{2}^{2}}{4 r_{0}}\left(e^{r_{0}(T-t)}-e^{r_{0}\left(T+t-2 t_{2}\right)}\right), \\
A_{6}(t)= & \frac{\lambda_{1}^{2}}{2 \gamma b_{1}^{2}}\left(T-t_{2}\right)+\beta(2 \beta+1) \sigma^{2} \int_{t}^{T} B(\nu) \mathrm{d} \nu,
\end{aligned}
$$

with $B(t), d_{4}(t)$ and $A_{4}(t)$ are given by equations (B.20) and (B.33).

(3) If $\rho_{12}<0,-\left(\lambda_{1} / \gamma b_{1} b_{2} \rho_{12}\right)>1$ and $\mathrm{e}^{r_{0} T}<-\left(\lambda_{1} /\right.$ $\left.\gamma b_{1} b_{2} \rho_{12}\right)$, the optimal reinsurance strategies of the big and small companies are expressed as those in equation (B.20) and $H(t, s, x)$ is given in equation (B.36).

(4) If $\rho_{12}<0, \quad-\left(\lambda_{1} / \gamma b_{1} b_{2} \rho_{12}\right)>1$ and $e^{r_{0} T} \geq-$ $\left(\lambda_{1} / \gamma b_{1} b_{2} \rho_{12}\right)$, when $0 \leq t \leq t_{3}$, the optimal reinsurance strategies of the big and small companies are the same as those in equation (B.23), when $t_{3}<t \leq t_{2}$, $p_{1}^{*}(t)$ and $p_{2}^{*}(t)$ are expressed as those in equation (B.9) and when $t_{2}<t \leq T, p_{1}^{*}(t)$ and $p_{2}^{*}(t)$ are shown in equation (B.30), i.e.,

$$
\left(p_{1}^{*}(t), p_{2}^{*}(t)\right)= \begin{cases}(0,1), & 0 \leq t \leq t_{3}, \\ \left(\frac{\lambda_{1}}{\gamma b_{1}^{2}} e^{-r_{0}(T-t)}+\frac{b_{2} \rho_{12}}{b_{1}}, 1\right), & t_{3}<t \leq t_{2}, \\ \left(\frac{\lambda_{1}}{\gamma b_{1}^{2}} e^{-r_{0}(T-t)}, 0\right), & t_{2}<t \leq T .\end{cases}
$$

Through the above derivatives, we obtain 


$$
H(t, s, x)= \begin{cases}-\frac{1}{\gamma} e^{-\gamma\left[e^{r_{0}(T-t)}\left(x-d_{7}(t)\right)+A_{7}(t)+B(t) s^{-2 \beta}\right],} & 0 \leq t \leq t_{3}, \\ -\frac{1}{\gamma} e^{-\gamma\left[e^{r_{0}(T-t)}\left(x-d_{5}(t)\right)+A_{5}(t)+B(t) s^{-2 \beta}\right]}, & t_{3}<t \leq t_{2}, \\ -\frac{1}{\gamma} e^{-\gamma\left[e^{r_{0}(T-t)}\left(x-d_{4}(t)\right)+A_{4}(t)+B(t) s^{-2 \beta}\right]}, & t_{2}<t \leq T,\end{cases}
$$

where

$$
\begin{aligned}
d_{7}(t)= & -\frac{D}{r_{0}}\left(1-e^{-r_{0}(T-t)}\right)+\frac{\lambda_{2}}{r_{0}}\left(1-e^{-r_{0}\left(t_{2}-t\right)}\right) \\
& -\frac{\lambda_{1} b_{2} \rho_{12}}{r_{0} b_{1}}\left(e^{-r_{0}\left(t_{3}-t\right)}-e^{-r_{0}\left(t_{2}-t\right)}\right) \\
& +\frac{\gamma b_{2}^{2}}{4 r_{0}}\left(e^{r_{0}(T-t)}-e^{r_{0}\left(T+t-2 t_{2}\right)}\right) \\
& -\frac{\gamma b_{2}^{2} \rho_{12}^{2}}{4 r_{0}}\left(e^{r_{0}\left(T+t-2 t_{3}\right)}-e^{r_{0}\left(T+t-2 t_{2}\right)}\right), \\
A_{7}(t)= & \frac{\lambda_{1}^{2}}{2 \gamma b_{1}^{2}}\left(T-t_{3}\right)+\beta(2 \beta+1) \sigma^{2} \int_{t}^{T} B(\nu) \mathrm{d} \nu,
\end{aligned}
$$

$$
\begin{aligned}
\mathrm{d}\left(H^{u_{1}^{*}, u_{2}^{*}}(t)\right)^{2}= & 2 H^{u_{1}^{*}, u_{2}^{*}}(t)\left\{\left[H_{x}^{u_{1}^{*}, u_{2}^{*}} \pi^{*}(t) \sigma S^{\gamma_{1}}(t)+H_{s}^{u_{1}^{*}, u_{2}^{*}} \sigma S^{\beta+1}(t)\right] \mathrm{d} W_{3}(t)+H_{x}^{u_{1}^{*}, u_{2}^{*}} p_{1}^{*}(t) b_{1} \mathrm{~d} W_{1}(t)\right. \\
& \left.-H_{x}^{u_{1}^{*}, u_{2}^{*}} p_{2}^{*}(t) b_{2} \mathrm{~d} W_{2}(t)+\mathscr{A}_{1}^{u_{1}^{*}, u_{2}^{*}} H^{u_{1}^{*}, u_{2}^{*}}(t) \mathrm{d} t\right\}+\left\{\left(H_{x}^{u_{1}^{*}, u_{2}^{*}}\right)^{2}\left[\left(\pi^{*}(t)\right)^{2} \sigma^{2} S^{2 \beta}(t)+\left(p_{1}^{*}(t)\right)^{2} b_{1}^{2}+\left(p_{2}^{*}(t)\right)^{2} b_{2}^{2}\right]\right. \\
& \left.+\left(H_{s}^{u_{1}^{*}, u_{2}^{*}}\right)^{2} \sigma^{2} S^{2 \beta+2}(t)+2 H_{x}^{u_{1}^{*}, u_{2}^{*}} H_{s}^{u_{1}^{*}, u_{2}^{*}} \pi^{*}(t) \sigma^{2} S^{2 \beta+1}(t)\right\} \mathrm{d} t .
\end{aligned}
$$

Since $u_{1}^{*}, u_{2}^{*}$ are the optimal strategies of equations (20) and (21), $\mathscr{A}^{\pi^{*}} H^{u_{1}^{*}, u_{2}^{*}}(t, s, x)=0$. Putting the expressions of with $B(t), d_{4}(t), A_{4}(t), d_{5}(t)$, and $A_{5}(t)$ are given by equations (B.20), (B.33), and (B.39).

To proof Theorem 1, we first introduce a lemma. For convenience, denote $O:=[0,+\infty) \times[0,+\infty) \times[0,+\infty)$.

Lemma B.1. Take a sequence of bounded open sets $O_{1}, O_{2}$, $O_{3}, \ldots$, with $O_{i} \subset O_{i+1} \subset O, i=1,2, \ldots$ and $\cup_{i} O_{i}=O$. Let $\tau_{i}$ be the exiting time of $\left(X^{u_{1}^{*}, u_{2}^{*}}(t), S(t)\right)$ from $O_{i}$. If one of the conditions (a) and (b) in Theorem 1 holds, then we have $E\left[H^{u_{1}^{*}, u_{2}^{*}}\left(\tau_{i} \wedge T, S\left(\tau_{i} \wedge T\right), X^{u_{1}^{*}, u_{2}^{*}}\left(\tau_{i} \wedge T\right)\right)^{2} \mid S(t)=\right.$ $\left.s, X^{u_{1}^{*}, u_{2}^{*}}(t)=x\right]<\infty$ for $i=1,2, \ldots$

Proof. We first denote $H^{u_{1}^{*}, u_{2}^{*}}(t)=H^{u_{1}^{*}, u_{2}^{*}}\left(t, S(t), X^{u_{1}^{*}, u_{2}^{*}}(t)\right)$ for simplicity. Applying Itô's formula, we have
$H_{x}, H_{s}, \pi^{*}(t), p_{1}^{*}(t)$, and $p_{2}^{*}(t)$ into equation (B.44), we obtain

$$
\begin{aligned}
\frac{\mathrm{d}\left(H^{u_{1}^{*}, u_{2}^{*}}(t)\right)^{2}}{\left(H_{1}^{u_{1}^{*}, u_{2}^{*}}(t)\right)^{2}}= & -2\left[\frac{\mu-r_{0}}{\sigma} S^{-\beta}(t) \mathrm{d} W_{3}(t)+\gamma \mathrm{e}^{r_{0}(T-t)} p_{1}^{*}(t) b_{1} \mathrm{~d} W_{1}(t)-\gamma e^{r_{0}(T-t)} p_{2}^{*}(t) b_{2} \mathrm{~d} W_{2}(t)\right] \\
& +\left[\frac{\left(\mu-r_{0}\right)^{2}}{\sigma^{2}} S^{-2 \beta}(t)+\gamma^{2} e^{2 r_{0}(T-t)}\left(p_{1}^{*}(t)\right)^{2} b_{1}^{2}+\gamma^{2} e^{2 r_{0}(T-t)}\left(p_{2}^{*}(t)\right)^{2} b_{2}^{2}\right] \mathrm{d} t .
\end{aligned}
$$


The solution to the above equation is

$$
\begin{aligned}
\frac{\left(H^{u_{1}^{*}, u_{2}^{*}}(t)\right)^{2}}{\left(H^{u_{1}^{*}, u_{2}^{*}}(0)\right)^{2}}= & \exp \left\{\int_{0}^{t}-\frac{2\left(\mu-r_{0}\right)}{\sigma} S^{-\beta}(\nu) \mathrm{d} W_{3}(\nu)-\frac{1}{2} \int_{0}^{t} \frac{4\left(\mu-r_{0}\right)^{2}}{\sigma^{2}} S^{-2 \beta}(\nu) \mathrm{d} \nu\right. \\
& +\int_{0}^{t}-2 \gamma e^{r_{0}(T-\nu)} p_{1}^{*}(\nu) b_{1} d W_{1}(\nu)-\frac{1}{2} \int_{0}^{t} 4 \gamma^{2} e^{2 r_{0}(T-\nu)}\left(p_{1}^{*}(\nu)\right)^{2} b_{1}^{2} \mathrm{~d} \nu \\
& +\int_{0}^{t} 2 \gamma e^{r_{0}(T-\nu)} p_{2}^{*}(\nu) b_{2} d W_{2}(\nu)-\frac{1}{2} \int_{0}^{t} 4 \gamma^{2} e^{2 r_{0}(T-\nu)}\left(p_{2}^{*}(\nu)\right)^{2} b_{2}^{2} \mathrm{~d} \nu \\
& \left.+\int_{0}^{t}\left(3 \gamma^{2} e^{2 r_{0}(T-\nu)}\left(p_{1}^{*}(\nu)\right)^{2} b_{1}^{2}+3 \gamma^{2} e^{2 r_{0}(T-\nu)}\left(p_{2}^{*}(\nu)\right)^{2} b_{2}^{2}+\frac{3\left(\mu-r_{0}\right)^{2}}{\sigma^{2}} S^{-2 \beta}(\nu)\right) \mathrm{d} v\right\} .
\end{aligned}
$$

According to the expression of the CEV model and Itô's is martingales and formula, we can derive

$\mathrm{d} S(t)^{-2 \beta}=\left(\beta(2 \beta+1) \sigma^{2}-2 \beta \mu S^{-2 \beta}(t)\right) \mathrm{d} t-2 \beta \sigma \sqrt{S^{-2 \beta}(t)} \mathrm{d} W_{3}(t)$.

$$
E\left[\exp \left\{\int_{0}^{t} \frac{3\left(\mu-r_{0}\right)^{2}}{\sigma^{2}} S^{-2 \beta}(\nu) \mathrm{d} \nu\right\}\right]<\infty,
$$

when one of the conditions (a) and (b) in Theorem 1 is satisfied. According to the expression of $p_{1}^{*}(t), p_{2}^{*}(t)$, and Novikov's condition,

By Zeng and Taksar [32], we know that

$\exp \left\{\int_{0}^{t}-\frac{2\left(\mu-r_{0}\right)}{\sigma} S^{-\beta}(\nu) \mathrm{d} W_{3}(\nu)-\frac{1}{2} \int_{0}^{t} \frac{4\left(\mu-r_{0}\right)^{2}}{\sigma^{2}} S^{-2 \beta}(\nu) \mathrm{d} \nu\right\}$,

$$
\begin{aligned}
& \exp \left\{\int_{0}^{t}-2 \gamma e^{r_{0}(T-\nu)} p_{1}^{*}(\nu) b_{1} \mathrm{~d} W_{1}(\nu)-\frac{1}{2} \int_{0}^{t} 4 \gamma^{2} e^{2 r_{0}(T-\nu)}\left(p_{1}^{*}(\nu)\right)^{2} b_{1}^{2} \mathrm{~d} v\right\}, \\
& \exp \left\{\int_{0}^{t} 2 \gamma e^{r_{0}(T-\nu)} p_{2}^{*}(\nu) b_{2} \mathrm{~d} W_{2}(\nu)-\frac{1}{2} 4 \int_{0}^{t} 2 \gamma e^{r_{0}(T-\nu)} p_{2}^{*}(\nu) b_{2}^{2} \mathrm{~d} \nu\right\},
\end{aligned}
$$

are martingales. Taking expectation from both sides of equation (B.46) yields

$$
\begin{aligned}
E\left[\left(H^{u_{1}^{*}, u_{2}^{*}}(t)\right)^{2}\right]= & \left(H^{u_{1}^{*}, u_{2}^{*}}(0)\right)^{2}\left[E \operatorname { e x p } \left\{\int_{0}^{t} 3 \gamma^{2} \mathrm{e}^{2 r_{0}(T-\nu)}\right.\right. \\
& \cdot\left(p_{1}^{*}(\nu)\right)^{2} b_{1}^{2}+3 \gamma^{2} \mathrm{e}^{2 r_{0}(T-\nu)}\left(p_{2}^{*}(\nu)\right)^{2} b_{2}^{2} \\
& \left.\left.+\frac{3\left(\mu-r_{0}\right)^{2}}{\sigma^{2}} S^{-2 \beta}(\nu) \mathrm{d} \nu\right\}\right]<\infty,
\end{aligned}
$$

i.e., $\quad E\left[\left(H^{u_{1}^{*}, u_{2}^{*}}\left(t, S(t), X^{u_{1}^{*}, u_{2}^{*}}(t)\right)\right)^{2}\right]<\infty$. So $E\left[\left(H^{u_{1}^{*}, u_{2}^{*}}\right.\right.$ $\left.\left(\tau_{i} \wedge T, S\left(\tau_{i} \wedge T\right), X^{u_{1}^{*}, u_{2}^{*}}\left(\tau_{i} \wedge T\right)\right)\right)^{2} \mid S(t)=s, X^{u_{1}^{*}, u_{2}^{*}}(t)=$ $x]<\infty$ for $i=1,2, \ldots$..

\section{Data Availability}

The data used to support the findings of this study are included within the article.

\section{Conflicts of Interest}

The authors declare that they have no conflicts of interest.

\section{Acknowledgments}

This research was supported by the National Natural Science Foundation of China (Nos. 11801179, 71573110, 71771220, 11971172, 71701084), the "Chenguang Program”, Shanghai, China (No. 18CG26), the Fundamental Research Funds for the Central Universities (No. 2019ECNU-HWFW028), and the 111 Project, China (No. B14019).

\section{References}

[1] H. Schmidli, "On minimizing the ruin probability by investment and reinsurance," The Annals of Applied Probability, vol. 12, no. 3, pp. 890-907, 2002.

[2] D. S. Promislow and V. R. Young, "Minimizing the probability of ruin when claims follow Brownian motion with drift," North American Actuarial Journal, vol. 9, no. 3, pp. 109-128, 2005. 
[3] L. Bai and J. Guo, "Optimal proportional reinsurance and investment with multiple risky assets and no-shorting constraint," Insurance: Mathematics and Economics, vol. 42, no. 3, pp. 968-975, 2008.

[4] H. Yang and L. Zhang, "Optimal investment for insurer with jump-diffusion risk process," Insurance: Mathematics and Economics, vol. 37, no. 3, pp. 615-634, 2005.

[5] N. Wang, "Optimal investment for an insurer with exponential utility preference," Insurance: Mathematics and Economics, vol. 40, no. 1, pp. 77-84, 2007.

[6] Y. Cao and N. Wan, "Optimal proportional reinsurance and investment based on Hamilton-Jacobi-Bellman equation," Insurance: Mathematics and Economics, vol. 45, no. 2, pp. 157-162, 2009.

[7] W. Yu, Y. Yong, G. Guan, Y. Huang, W. Su, and C. Cui, "Valuing guaranteed minimum death benefits by cosine series expansion," Mathematics, vol. 7, no. 9, p. 835, 2019.

[8] Z. Zhang, Y. Yong, and W. Yu, "Valuing equity-linked dealth benefits in general exponential Lévy models," Journal of Computational and Applied Mathematics, vol. 365, Article ID 112377, 2020.

[9] X. Peng, W. Su, and Z. Zhang, "On a perturbed compound Poisson risk model under a periodic threshold-type dividend strategy," Journal of Industrial and Management Optimization, vol. 13, no. 5, pp. 1-20, 2019.

[10] W. Yu, F. Wang, Y. Huan, and H. Liu, "Social optimal mean field control problem for population growth model," Asian Journal of Control, 2019.

[11] J. Ruan, W. Yu, K. Song, Y. Sun, Y. Huang, and X. Yu, “A note on a generalized Berber-Shiu discounted penalty function for a compound Poisson risk model," Mathematics, vol. 7, no. 10, p. 891, 2019.

[12] W. Yu, Y. Huan, and C. Cui, "The absolute ruin insurance risk model with a threshold dividend strategy," Symmetry, vol. 10, no. 9, p. 377, 2018.

[13] Y. Huang, W. Yu, Y. Pan, and C. Cui, "Estimating the gerbershiu expected discounted penalty function for lévy risk model," Discrete Dynamics in Nature and Society, vol. 2019, Article ID 3607201, 15 pages, 2019.

[14] Y. Zeng, D. Li, and A. Gu, "Robust equilibrium reinsuranceinvestment strategy for a mean-variance insurer in a model with jumps," Insurance: Mathematics and Economics, vol. 66, pp. 138-152, 2016.

[15] D. Li, Y. Zeng, and H. Yang, "Robust optimal excess-of-loss reinsurance and investment strategy for an insurer in a model with jumps," Scandinavian Actuarial Journal, vol. 2018, no. 2, pp. 145-171, 2018.

[16] J. Suijs, A. De Waegenaere, and P. Borm, "Stochastic cooperative games in insurance," Insurance: Mathematics and Economics, vol. 22, no. 3, pp. 209-228, 1998.

[17] X. Zeng, "A stochastic differential reinsurance game," Journal of Applied Probability, vol. 47, no. 2, pp. 335-349, 2010.

[18] M. Taksar and X. Zeng, "Optimal non-proportional reinsurance control and stochastic differential games," Insurance: Mathematics and Economics, vol. 48, no. 1, pp. 64-71, 2011.

[19] A. Bensoussan, C. C. Siu, S. C. P. Yam, and H. Yang, "A class of non-zero-sum stochastic differential investment and reinsurance games," Automatica, vol. 50, no. 8, pp. 2025-2037, 2014.

[20] H. Meng, S. Li, and Z. Jin, "A reinsurance game between two insurance companies with nonlinear risk processes," Insurance: Mathematics and Economics, vol. 62, pp. 91-97, 2015.
[21] C. S. Pun and H. Y. Wong, "Robust non-zero-sum stochastic differential reinsurance game," Insurance: Mathematics and Economics, vol. 68, pp. 169-177, 2016.

[22] C. C. Siu, S. C. P. Yam, H. Yang, and H. Zhao, "A class of nonzero-sum investment and reinsurance games subject to systematic risks," Scandinavian Actuarial Journal, vol. 2017, no. 8, pp. 670-707, 2017.

[23] C. J. Cox and S. A. Ross, "The valuation of options for alternative stochastic processes," Journal of Financial Economics, vol. 4, no. 1-2, pp. 145-166, 1976.

[24] E. M. Stein and J. C. Stein, "Stock price distributions with stochastic volatility: an analytic approach," Review of Financial Studies, vol. 4, no. 4, pp. 727-752, 1991.

[25] S. L. Heston, "A closed-form solution for options with stochastic volatility with applications to bond and currency options," Review of Financial Studies, vol. 6, no. 2, pp. 327343, 1993.

[26] M. Gu, Y. Yang, S. Li, and J. Zhang, "Constant elasticity of variance model for proportional reinsurance and investment strategies," Insurance: Mathematics and Economics, vol. 46, no. 3, pp. 580-587, 2010.

[27] Z. Liang, K. C. Yuen, and K. C. Cheung, "Optimal reinsurance-investment problem in a constant elasticity of variance stock market for jump-diffusion risk model," Applied Stochastic Models in Business and Industry, vol. 28, no. 6, pp. 585-597, 2012.

[28] X. Lin and Y. Li, "Optimal reinsurance and investment for a jump diffusion risk process under the CEV model," North American Actuarial Journal, vol. 15, no. 3, pp. 417-431, 2011.

[29] A. Gu, X. Guo, Z. Li, and Y. Zeng, "Optimal control of excessof-loss reinsurance and investment for insurers under a CEV model," Insurance: Mathematics and Economics, vol. 51, no. 3, pp. 674-684, 2012.

[30] X. Zheng, J. Zhou, and Z. Sun, "Robust optimal portfolio and proportional reinsurance for an insurer under a CEV model," Insurance: Mathematics and Economics, vol. 67, pp. 77-87, 2016.

[31] J. C. Cox, "The constant elasticity of variance option pricing model," The Journal of Portfolio Management, vol. 23, no. 5, pp. 15-17, 1996.

[32] X. Zeng and M. Taksar, "A stochastic volatility model and optimal portfolio selection," Quantitative Finance, vol. 13, no. 10, pp. 1547-1558, 2013. 